\title{
Mô phỏng hierarchy MCMC cho mô hình Satlns end + ses + res + insured, BayesVL v0.6.5 trên 1042 quan sát thực
}

\author{
Vương Quân Hoàng \\ Lã Việt Phương
}

AISDL (Vuong \& Associates)

SDAG, Centre for Interdisciplinary Social Research (Phenikaa University)

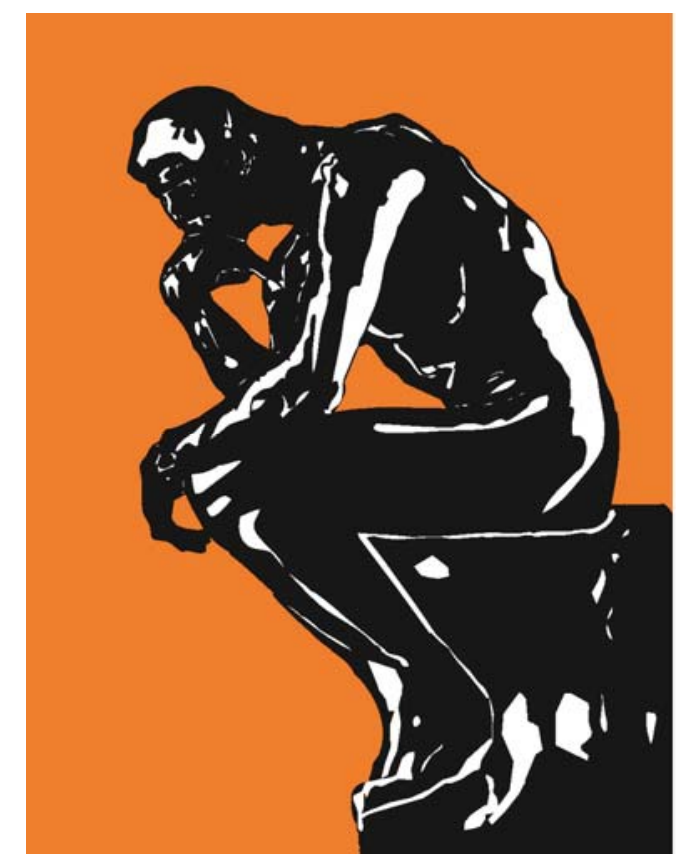

Note $2 b$

Hà Nội, 28-4-2019 (6:30 PM version 3)

Bài viết này (Note $\mathbf{2 b}$ ) trình bày một mô hình và ứng dụng mô phỏng BayesVL v0.6.5 [1] trên môi trường $R$ và Stan MCMC, cho bài toán thực tế [2] với dữ liệu thực tế, gồm 1042 điểm dữ liệu [3]. Nội dung của bài là một phần của tài liệu do AISDL biên soạn phục vụ hướng dẫn sử dụng "The BayesVL R Package", nhằm thúc đẩy việc sử dụng phương pháp thống kê Bayesian trong KHXH\&NV.

Một phần mã chương trình [1] được viết dựa trên Statistical Rethinking của Richard McElreath [5] và BNLearn của Scuttari [6]. Công việc mô phỏng cơ bản sử dụng kỹ thuật Hamiltonian Markov chain Monte Carlo, dựa trên Stan và R.

\section{Bài toán cần xử lý}

Bài toán sử dụng cơ sở dữ liệu "Health Care, Medical Insurance, and Economic Destitution: A Dataset of 1042 Stories" [3].

*Công việc: Tiến hành lại việc đánh giá áp lực kinh kế lên bệnh nhân như thế nào khi có hoặc không có nhà ở và bảo hiểm y tế, dựa trên mô phỏng $\mathrm{MCMC}$ thay cho ước 
lượng frequentist như trong [2], tương tự như với mô hình [4] nhưng mở rộng và cũng phức tạp hơn do tính chất "nested multi-level" (hierarchy model).

2. Dữ liệu và đánh giá mô hình (dataset and estimations)

a. Dữ liệu và xây dựng mô hình

data1<-read.csv("/Statistics/1042/1042data/1042data.csv", header = TRUE) head(data1)

Các biến sử dụng:

- Res: bệnh nhân có phải là cư dân ở khu vực khám chữa bệnh không

- Insured: bệnh nhân có chế độ bảo hiểm không

- SES : tình trạng kinh tế của bệnh nhân

- End: kết thúc quá trình điều trị

- SatIns: mức độ hài lòng của bệnh nhân

Tương tự bài toán $2 a$, ta dựng mô hình quan hệ giữa các biến dạng lưới như sau:

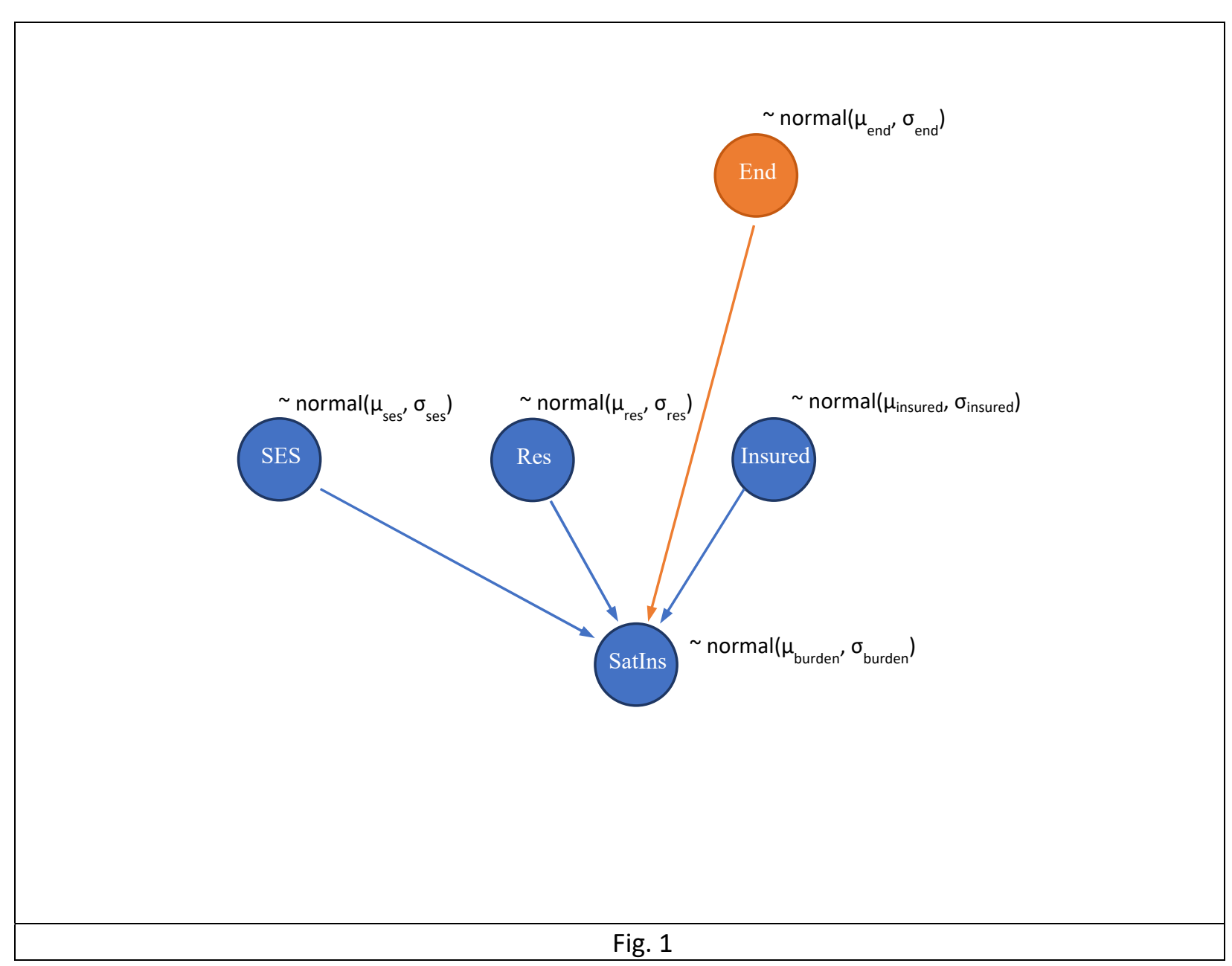

Sử dụng packages bayesvl trên R mô tả mô hình quan hệ ở trên: 


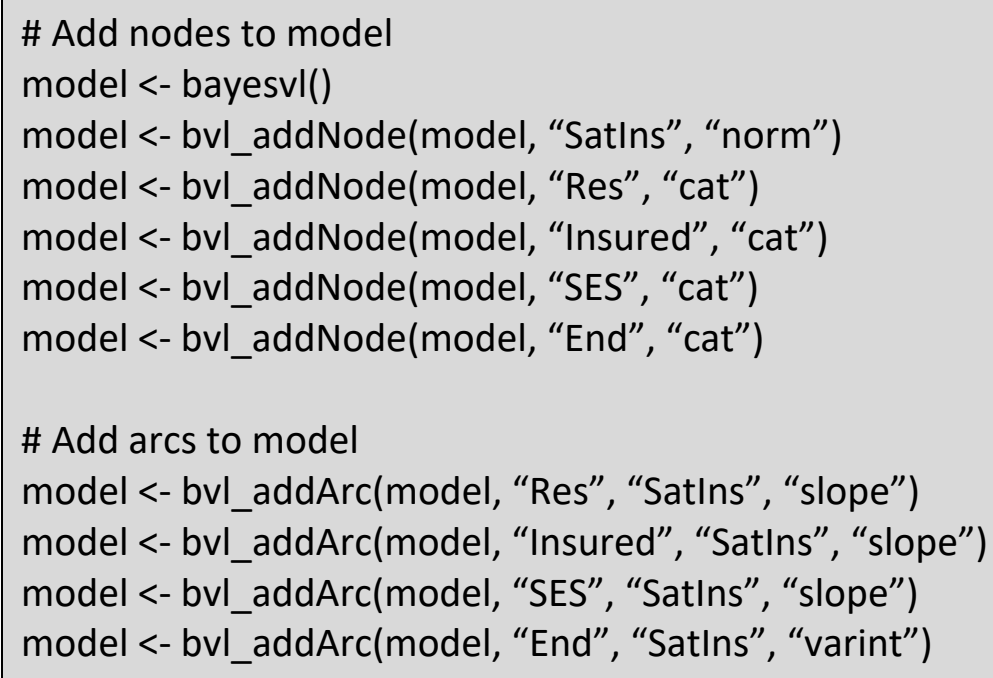

So với mô hình trong Note 2a [4], ta đã thêm 2 nodes cho 2 biến SES và End vào phương trình mô phỏng. Cần đặc biệt chú rằng, biến End có quan hệ với Burden thông qua arc loại varint chứ không phải slope.

Đây là loại quan hệ phân lớp multi-level dạng varying intercept.

\section{Mô hình multi-level:}

Nếu như trên mô hình hồi quy đơn giản Note 2a, công thức toán học có dạng:

$$
y i=\alpha+\beta x i+\epsilon i
$$

Thì với mô hình đa lớp (multilevel) varying intercept công thức toán học của mô hình sẽ có dạng:

$$
y_{i}=\alpha_{j}[i]+\beta x i+\epsilon i
$$

Ví dụ mô hình quan hệ ở trên, nếu các nodes đều là kết nối slope với node Satlns, phương trình toán học mô hình sẽ có dạng:

$$
y i=\alpha j[i]+\beta 1 \times 1 i+\beta 2 \times 2 i+\beta 3 \times 3 i+\epsilon i
$$


Trong đó:

- y là biến outcome (Satlns), tức là response variable.

- x1, x2, x3 là các biến độc lập quan hệ slope tương ứng Res, Insured và SES.

- $\quad \alpha$ là hệ số varying intercept của End với $\mathrm{j}=1,2,3,4$, tương ứng các mức giá trị $A, B, C, D$ của biến End.

Minh họa các đường tuyến tính mô phỏng được sẽ dịch chuyển tùy theo alpha

So sánh 2 mô hình trên ta có thể thấy một số dạng thay đổi hệ số intercept alpha theo End như sau:

\section{Complete pooling:}

Tất cả các mức giá trị của biến End $(A, B, C, D)$ được đối xử như nhau khi hồi quy thường.

Có thể hiểu là các giá trị biến End được cho chung hết vào 1 túi khi thực hiện hồi quy với các biến khác (complete pooling).

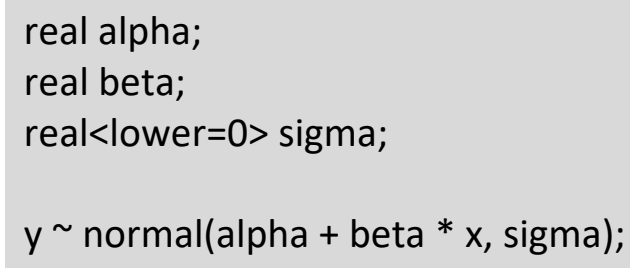

\section{No pooling:}


Ở mô hình multilevel, các giá trị của biến End được đánh giá độc lập khi hồi quy. Hay có thể hiểu dữ liệu được phân hoạch vào các "túi" khác nhau.

Như vậy việc chọn mô hình multilevel với biến End, ta đã giả định các bệnh nhân sẽ có mức độ hài lòng rất khác nhau ở các kết quả kết thúc điều trị khác nhau.

Bằng cách này mô hình cho phép chia sẻ sức mạnh trong từng mức giá trị của biến End, cho phép có kết quả suy luận hợp lý hơn ở các mức giá trị End có ít dữ liệu, và tính đại diện của mẫu khảo sát không tốt lắm.

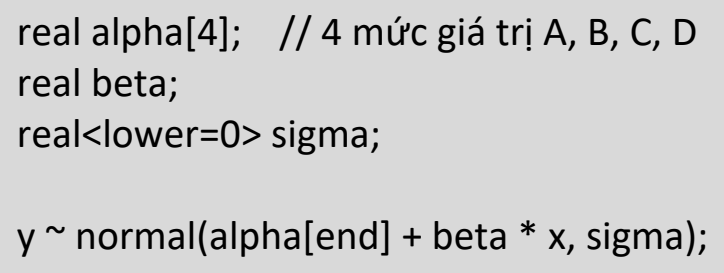

\section{Partial pooling:}

Mô hình không phân hoạch (complete pooling) và mô hình phân hoạch (no pooling) ở trên đều có hạn chế. Với mô hình hồi quy thường, giá trị apha sẽ sẽ được mô phỏng giao động quanh 1 giá trị trung bình mu và không phân hoạch theo các mức biến End.

Ở mô hình phân hoạch no-pooling, các giá trị alpha khác nhau sẽ được mô phỏng cho từng mức giá trị của biến End các giá trị của biến End được đánh giá độc lập khi hồi quy.

Tuy nhiên mô hình này cũng có vấn đề, với một số mức giá trị biến End có số mẫu dữ liệu ít hơn nhiều so với các mức khác có thể cho các mức giá trị alpha $(\alpha)$ cực đoan, ta cũng thường không tin tưởng nhiều nếu mức này chỉ có một vài quan sát (không đại diện tốt cho tập giá trị thực tế).

Mô hình partial pooling tương tự như mô hình đa cấp no pooling nhưng gộp một phần các mô phỏng vector hệ số alpha $\left(\alpha_{j}\right)$ về dao động quanh mức trung bình mu $(\mu)$ của chúng.

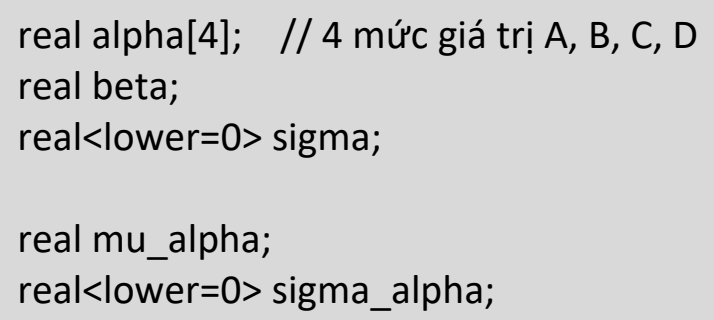




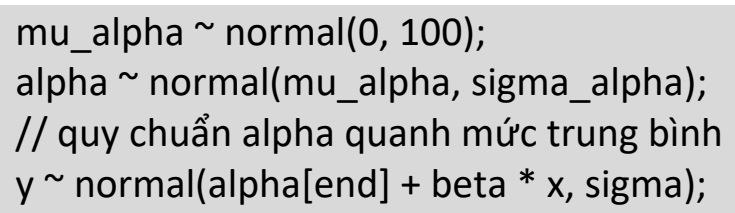

\section{Các dạng mô hình multi-level khác:}

Tương tự như vậy, với mô hình đa lớp (multilevel) varying slope công thức toán học của mô hình sẽ có dạng:

$$
y i=\alpha+\beta j[i] x i+\epsilon i
$$

Có thể nhận thấy lúc này hệ số góc $\beta(\beta)$ của biến độc lập $\mathrm{x}$ đã được thể hiện dưới dạng đa lớp.

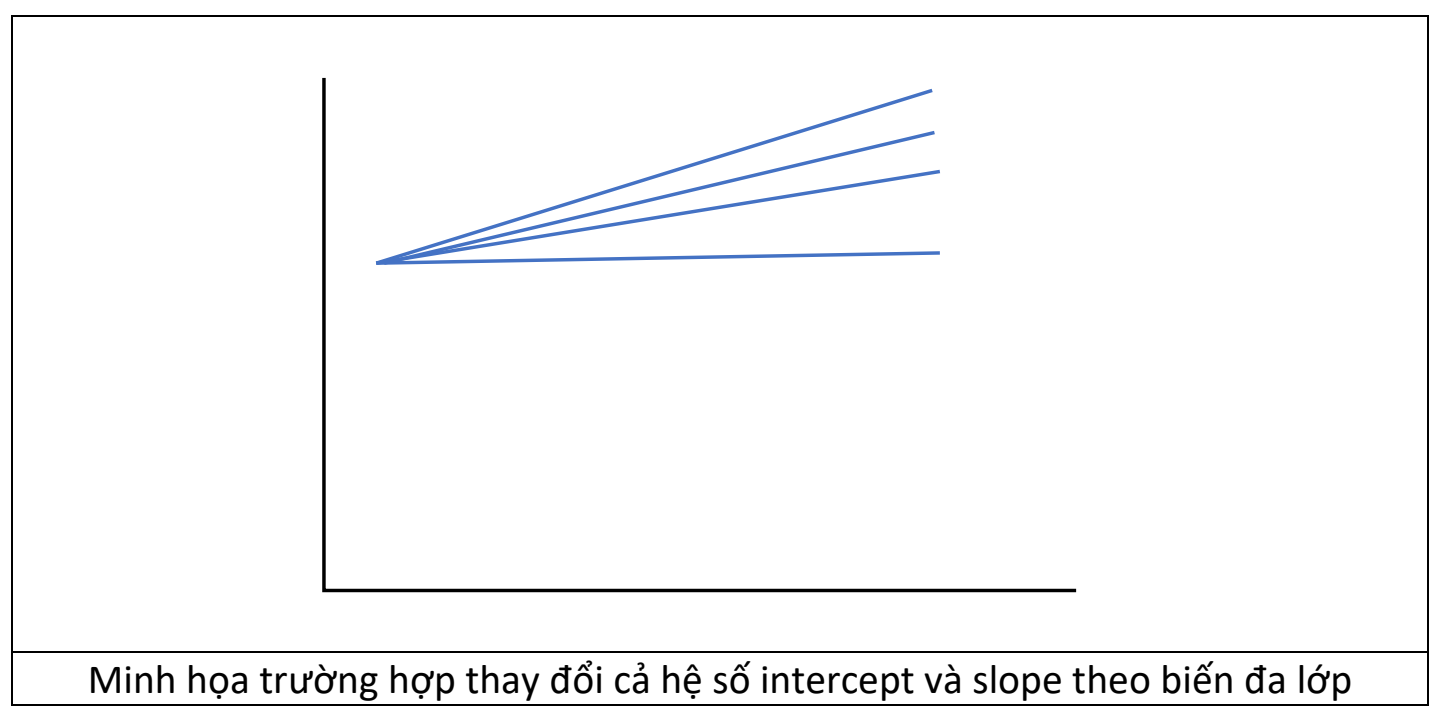

Khi thay đổi cả hệ số intercept $(\alpha)$ và slope $(\beta)$ theo biến đa lớp ta có mô hình varying intercept and varying slope có dạng:

$$
y_{i}=\alpha j[i]+\beta_{j}[i] x i+\epsilon i
$$

b. Đánh giá mô hình

- Đánh giá mô hình bằng bnlearn 
Tính xác suất có điều kiện các biến trong mô hình:

bvl_bnBayes(model, data1[c("Res","SatIns","Insured", "SES","End")])

Kết quả:

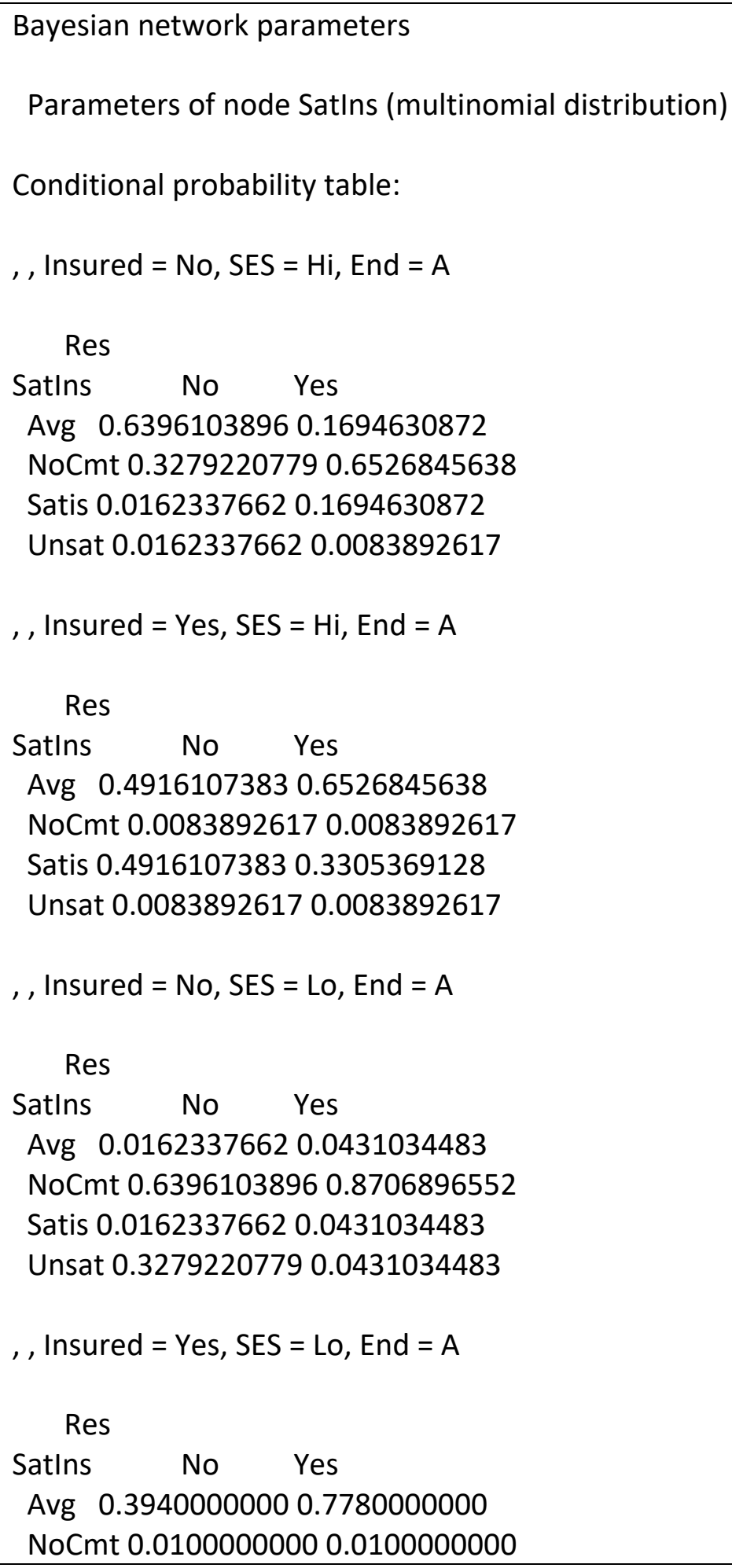




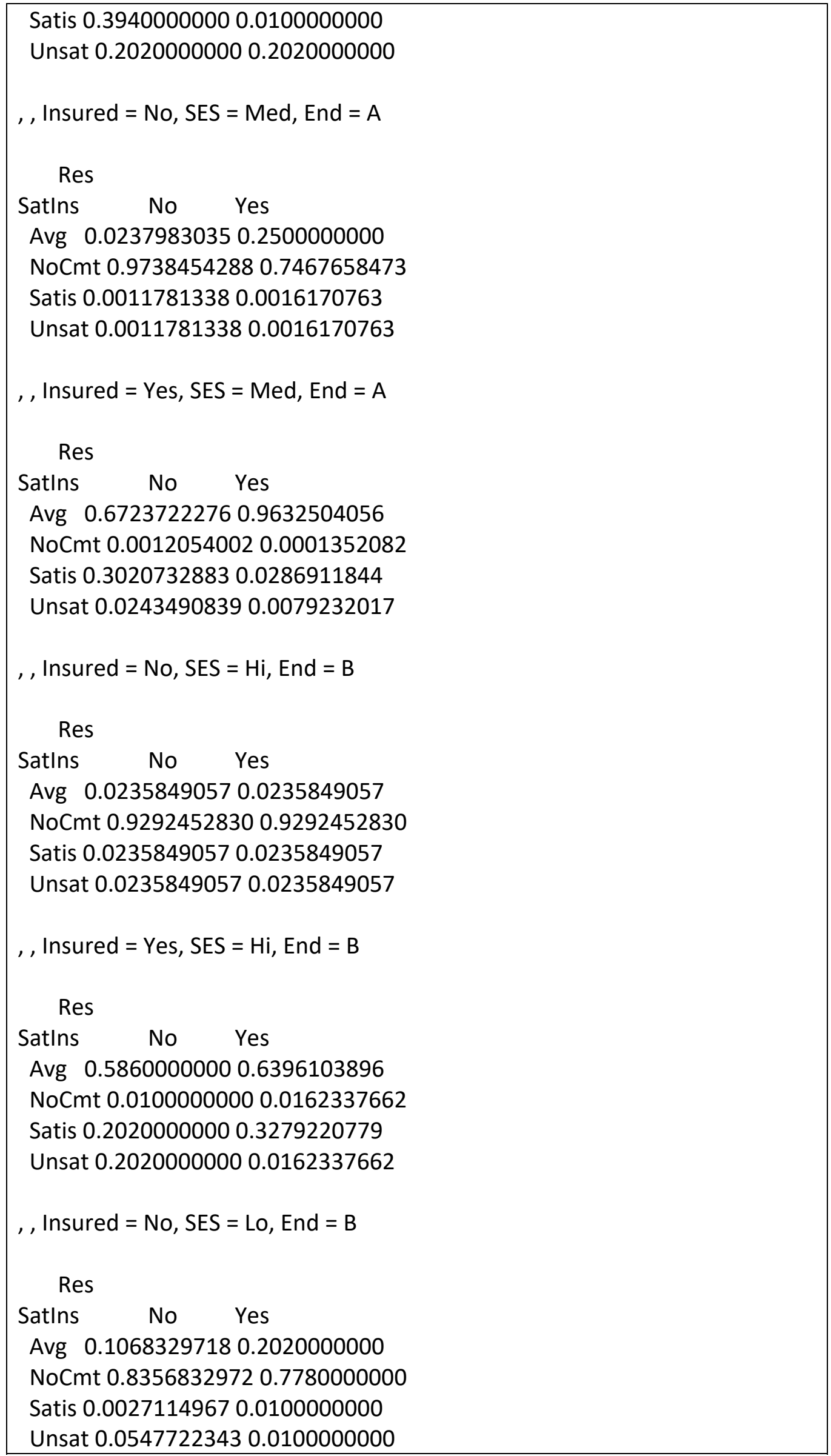




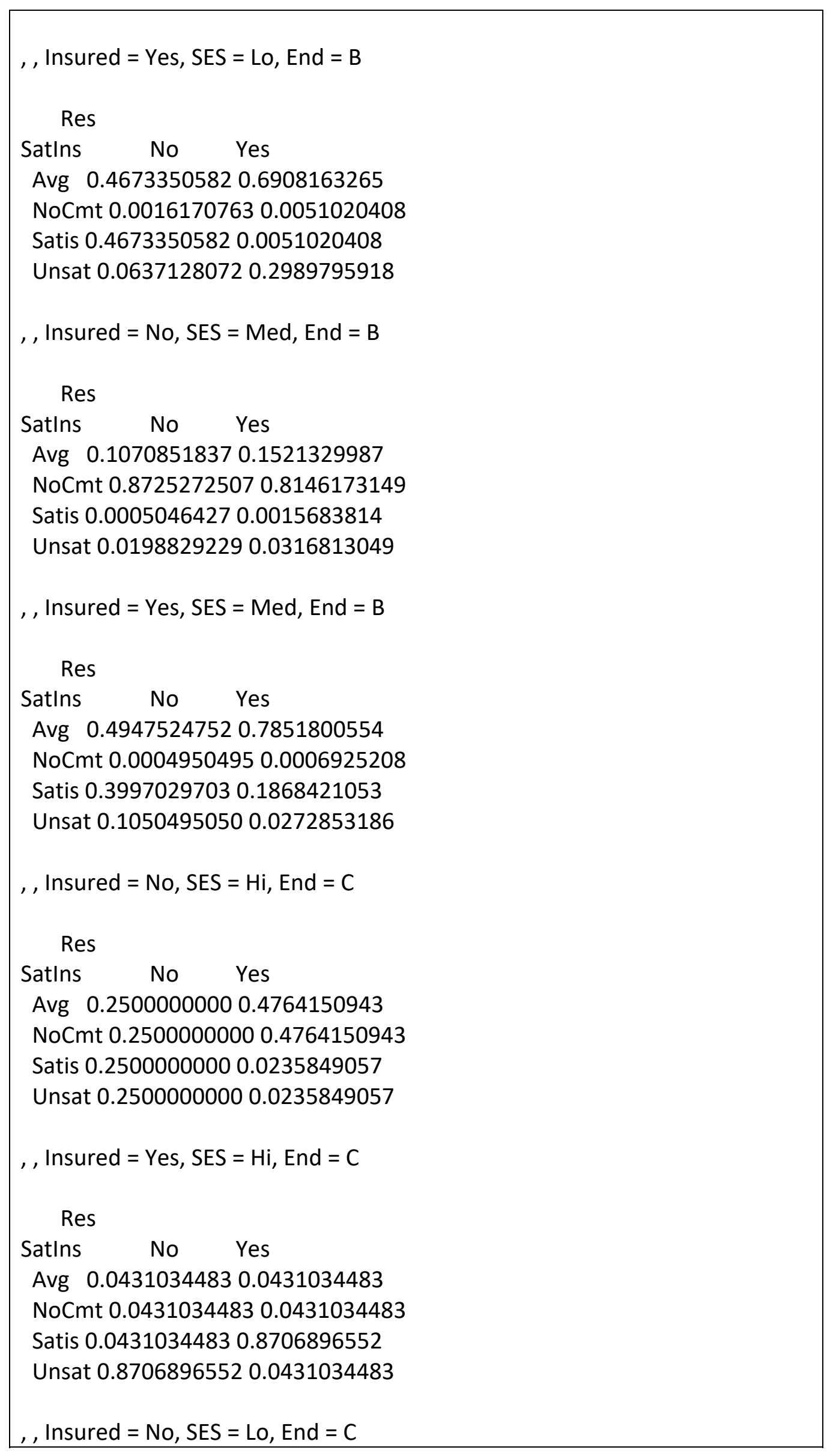




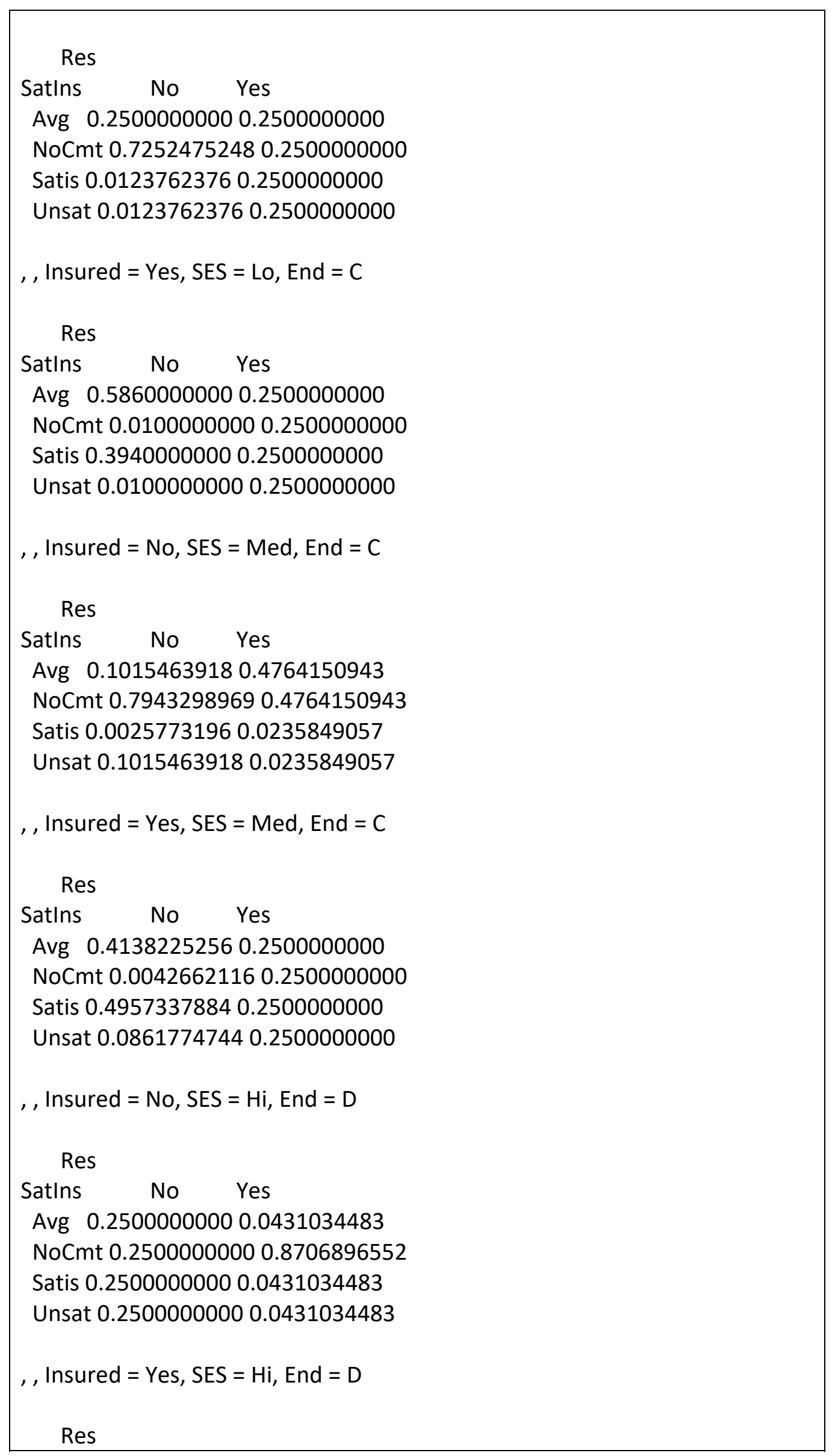




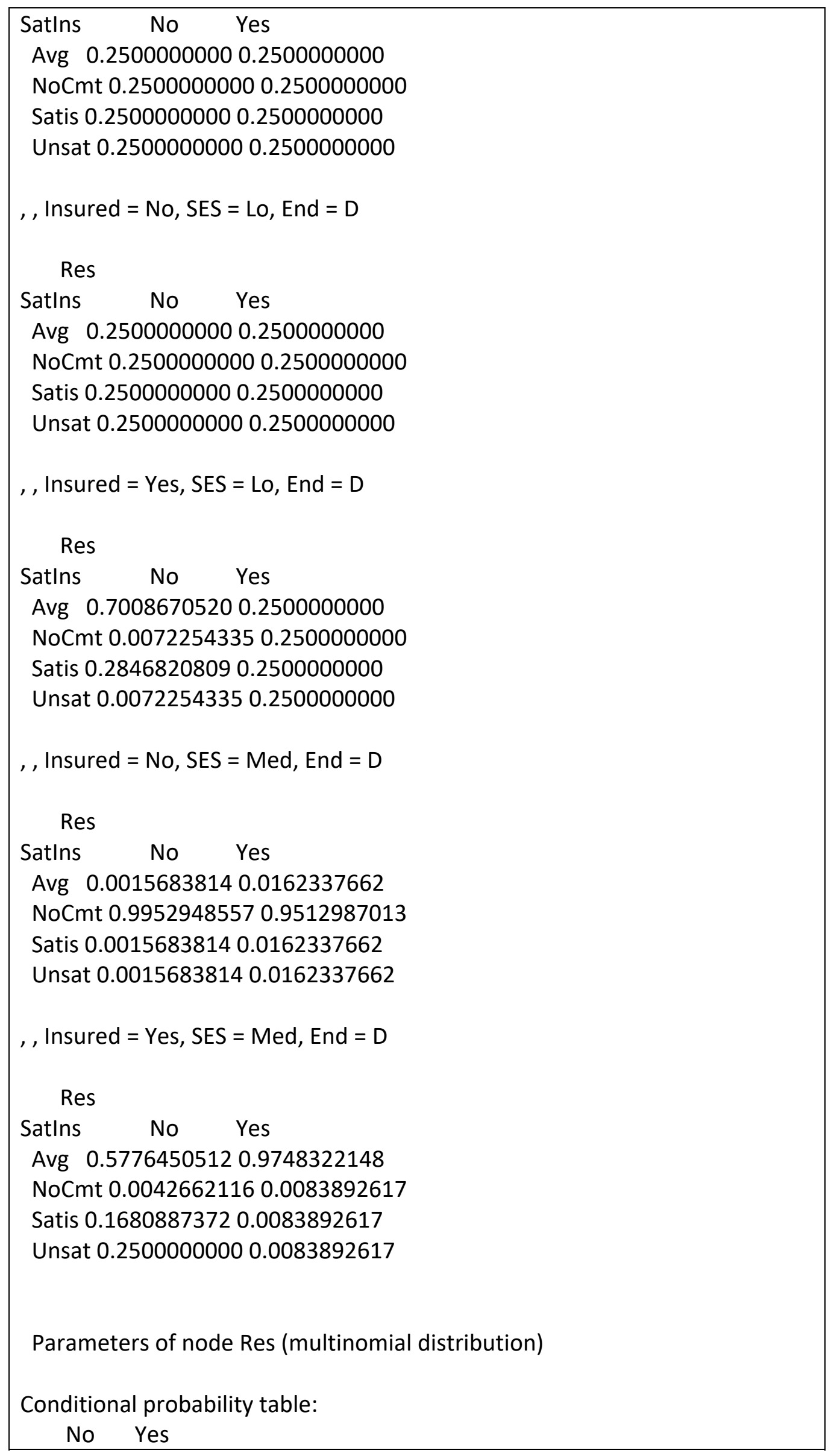




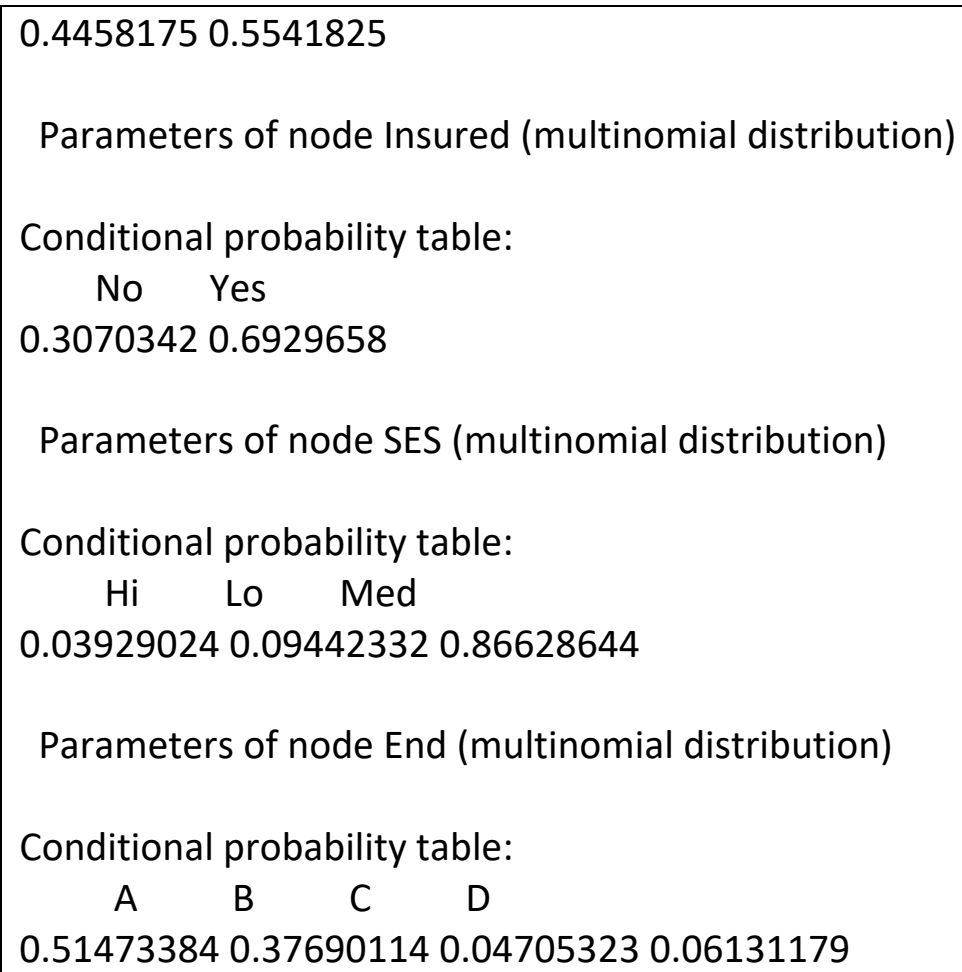

Nếu vẽ các xác suất có điều kiện này:

bvl_bnBarchart(model, data1[c("Res","SatIns","Insured","SES","End")])

Ta sẽ thu được:

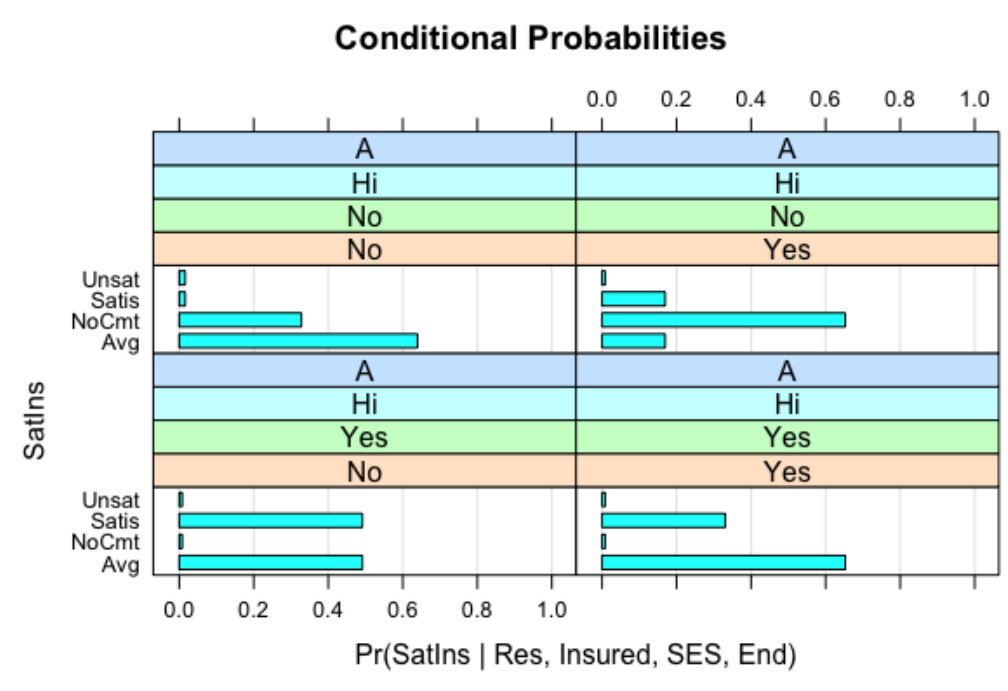




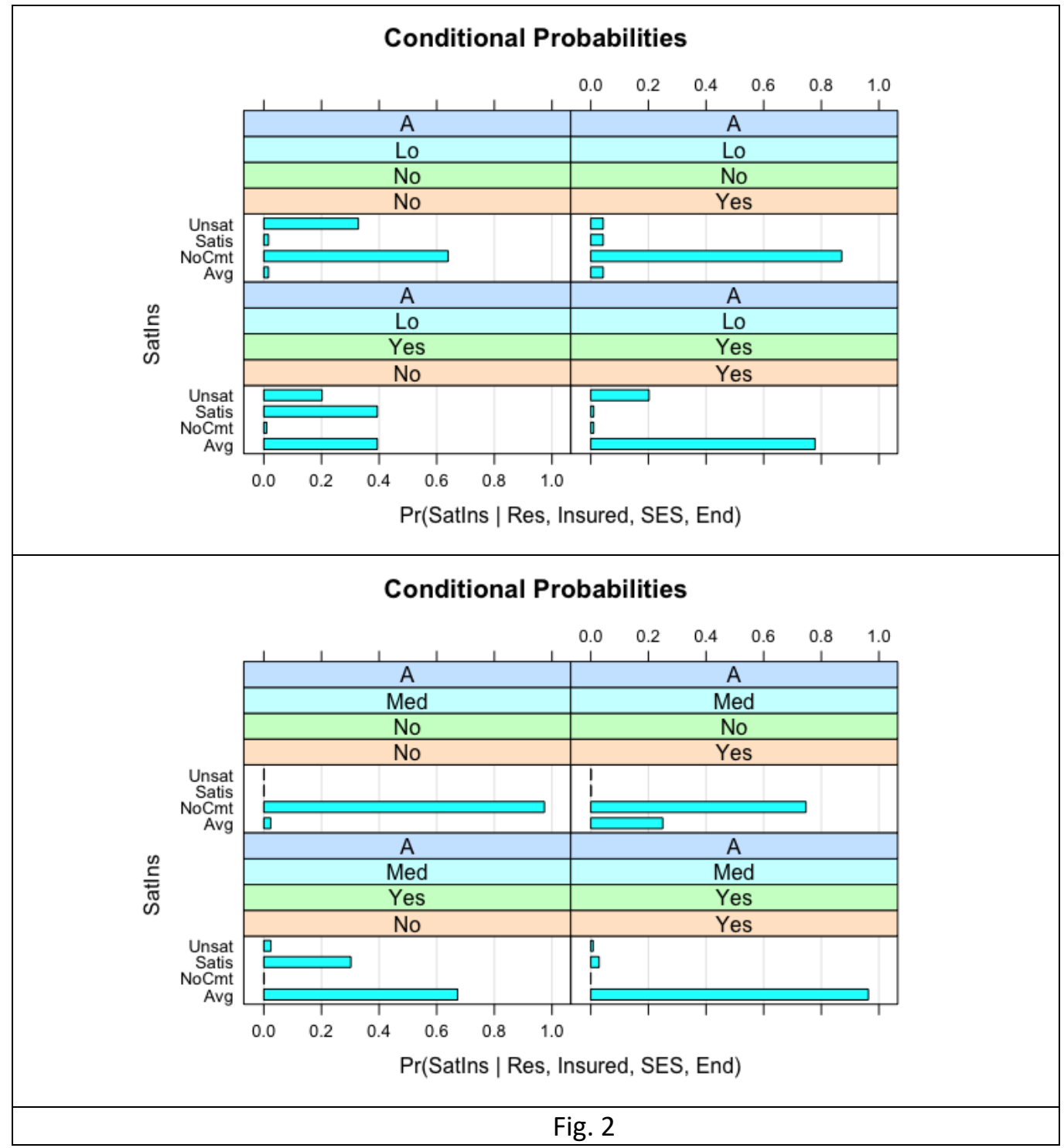

Đánh giá sức mạnh kết nối của mô hình quan hệ:

>bvl_bnStrength(model, data1[c("Res","SatIns","Insured","SES","End")]) from to strength

1 Res SatIns 5.314485e-03

2 Insured SatIns 4.063128e-125

3 SES SatIns 1.014490e-01

4 End SatIns 9.498561e-01

Chức năng này gọi package bnlearn để đo lường quan hệ giữa các biến trong lưới model thông qua xác suất tương ứng từng cung $(\operatorname{arc})$. Giá trị strength được thể hiện chính là giá trị p-value.

Mô hình hiện tại có 4 arc, các giá trị p-values đều nhỏ hơn 0,05 và support rất tốt từ dữ liệu. 


\section{c. Mô hình hồi quy bayesian}

Với mô hình quan hệ đã tạo ở trên, nếu viết ở dạng hồi quy tuyến tính, ta có phương trình:

$$
\mathrm{y}_{\text {satins }}[\mathrm{i}]=\alpha_{\text {end }}\left[\mathrm{x}_{\text {end }}[\mathrm{i}]\right]+\beta_{\text {res }} \mathrm{x}_{\text {res }}[\mathrm{i}]+\beta_{\text {insured }} \mathrm{x}_{\text {insured }}[\mathrm{i}]+\beta_{\text {ses }} \mathrm{x}_{\text {ses }}[\mathrm{i}]+\epsilon_{\text {satins }}[\mathrm{i}]
$$

Trên phương trình ta thấy có 3 hệ số $\beta_{\text {res, }} \beta_{\text {insured }}$ và $\beta_{\text {ses }}$ là 3 hệ số hồi quy "slope" là hệ số ảnh hưởng đến hệ số góc của đường hồi quy, 1 hệ số varying intercept là $\alpha_{\text {end. }}$. Khi tạo mô hình quan hệ các biến ở trên ta đã định nghĩa arc với loại quan hệ "slope":

$$
\begin{aligned}
& \text { model <- bvl_addArc(model, "Res", "Burden", "slope") } \\
& \text { model <- bvl_addArc(model, "Insured", "Burden", "slope") } \\
& \text { model <- bvl_addArc(model, "SES", "Burden", "slope") }
\end{aligned}
$$

Và quan hệ varying intercept

$$
\text { model <- bvl_addArc(model, "SES", "Burden", "varint") }
$$

Vì vậy, để tạo mô hình hồi quy trên bayesvl chỉ cần thực hiện lệnh:

$$
\begin{aligned}
& \text { \# Generate the stan code for model } \\
& \text { model_string <- bvl_model2Stan(model) } \\
& \text { cat(model_string) }
\end{aligned}
$$

Mô hình được tạo sẽ ở dạng no-pooling varying intercept with mixed effects

model_string chứa code stan cho mô hình hồi quy tuyến tính.

Code stan được tạo:

data\{

// Define variables in data

int<lower=1> Nobs; // Number of observations (an integer)

real SatIns[Nobs]; // outcome variable

int Res[Nobs];

int Insured[Nobs];

int SES[Nobs]; 


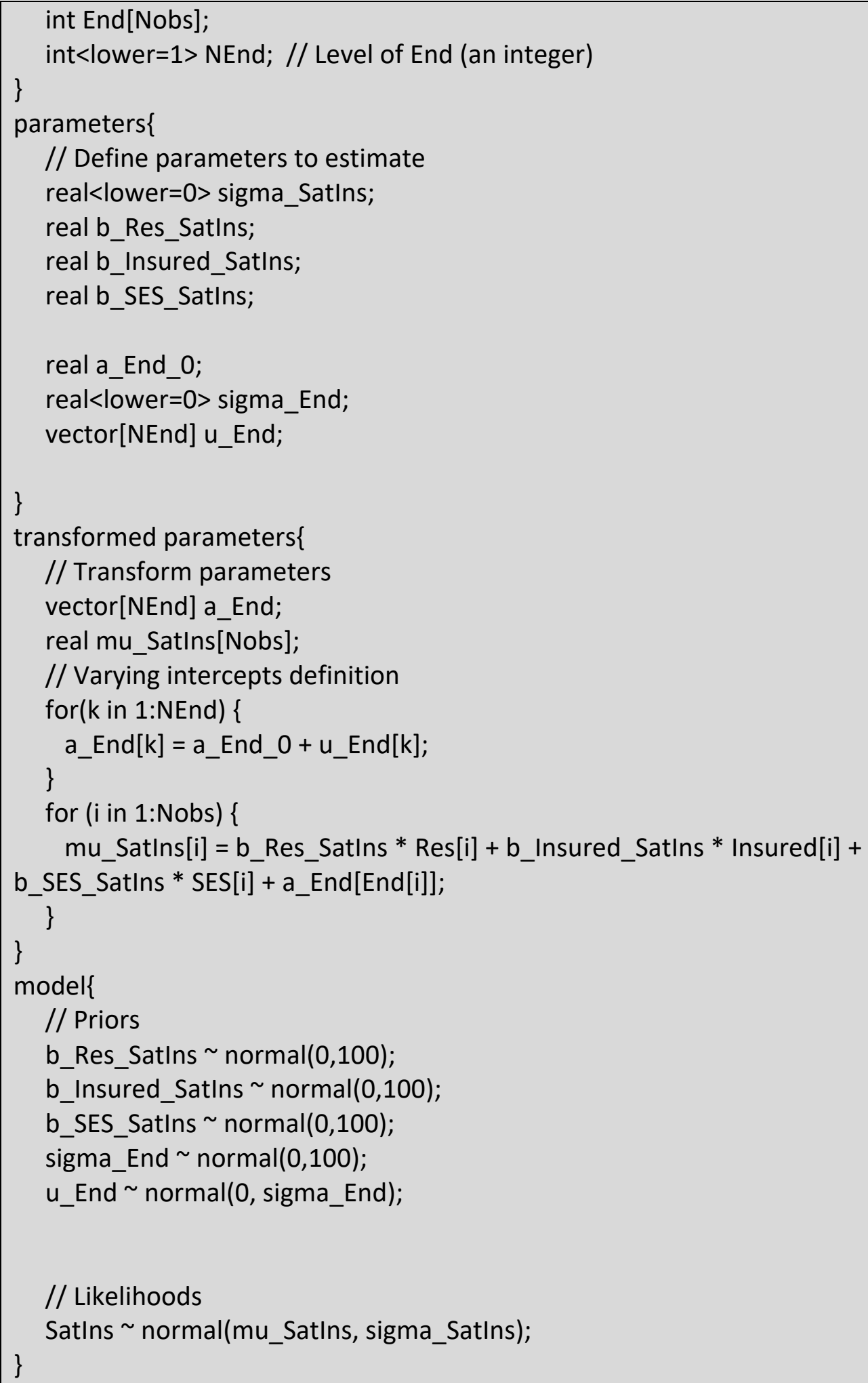

Chạy mô phỏng mô hình:

dat1042 <- with(data1, list(Nobs = length(Res), 


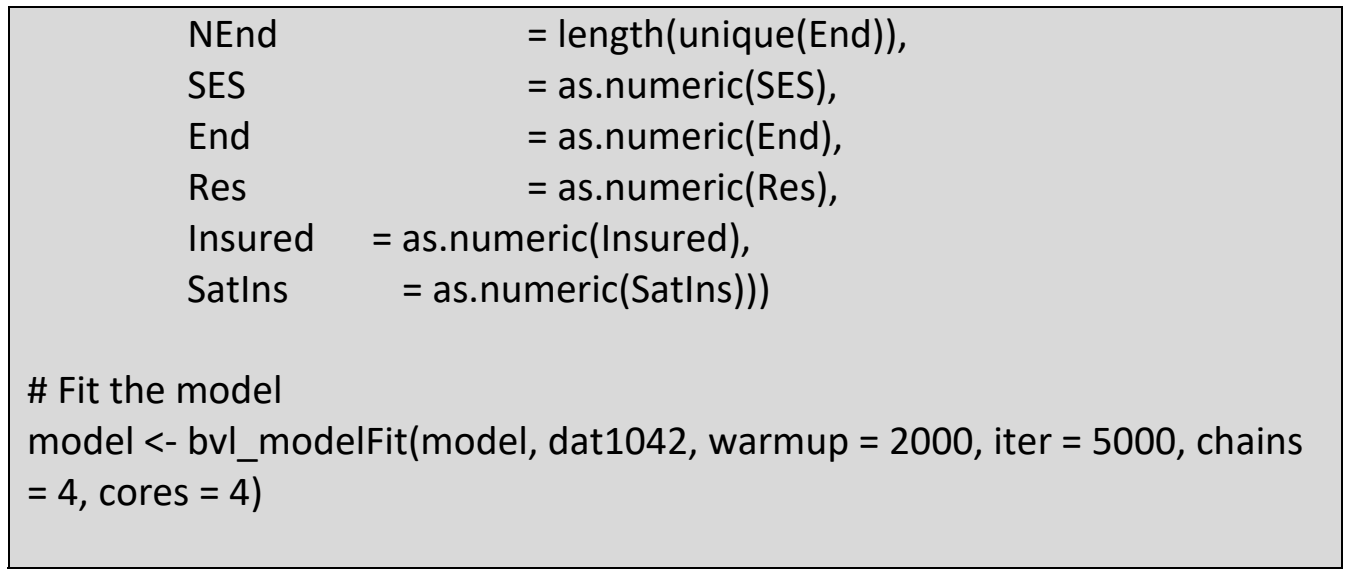

d. Đánh giá mô hình

Đánh giá sự hội tụ của mô hình có thể thực hiện thông qua trace plot:

$$
\text { traceplot(fit) }
$$

Đồ họa thu được trong Figure 3:

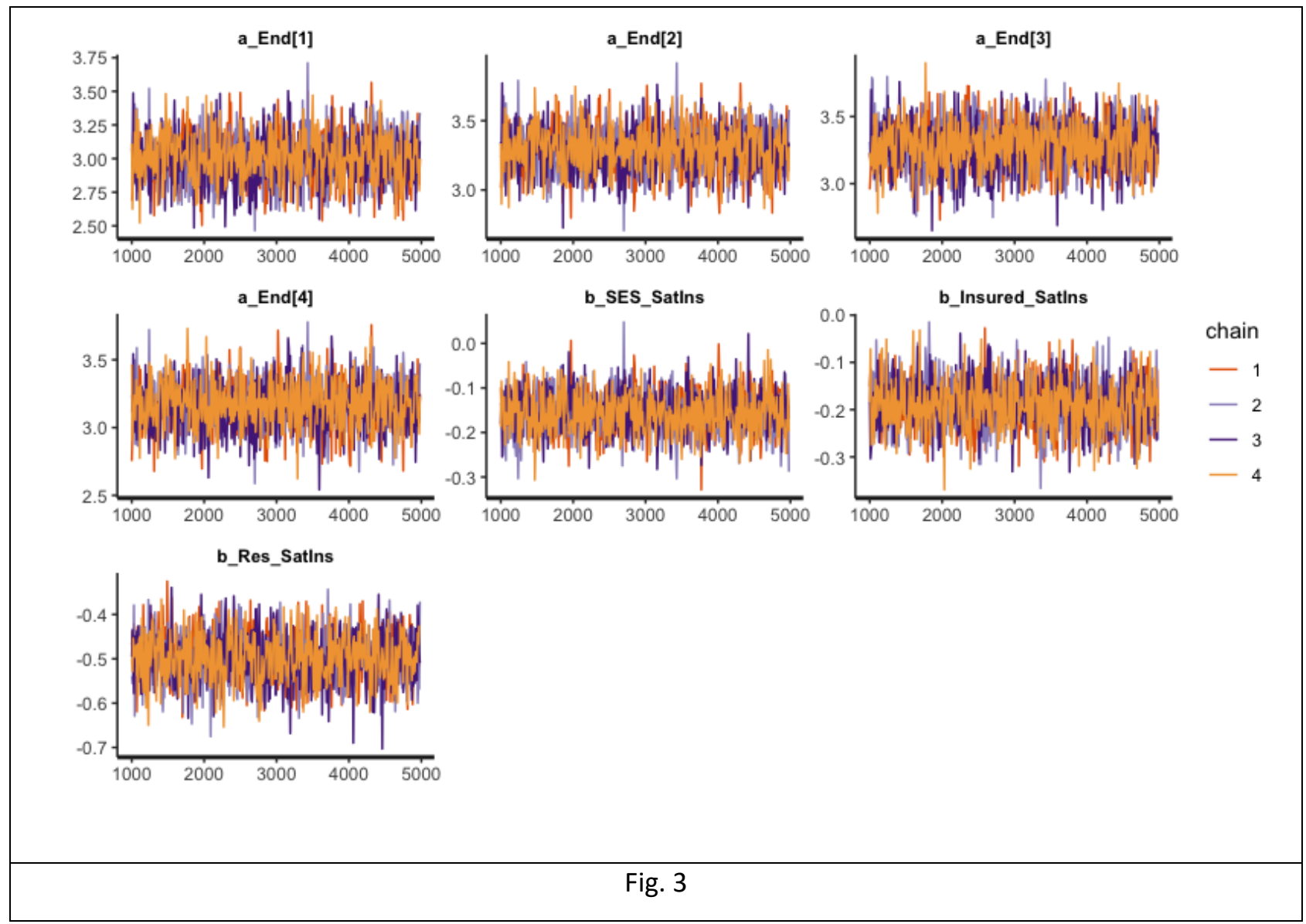

Ở mô hình này ta thấy trace plots tốt, không có các chuỗi dị thường divergent chains 
Bước tiếp theo ta có thể xem summary output.

summary(fit)

Cơ bản kết quả thu được khá tốt, đảm bảo quy cách kỹ thuật của mô phỏng:

> summary(fit)
Model Info:
Nodes: $\quad 3$
Arcs: $\quad 2$

Scores:

Formulas: $\quad$ SatIns $\sim a \_E n d[$ End $]+b \_R e s *$ Res $+b \_I n s u r e d *$ Insured $+b \_S E S *$ SES

Estimates:

4 chains, each with iter $=5000$; warmup $=1000$; thin $=10$;

$\begin{array}{lllllllllll}\text { a_End[1] } \quad 3.00 & 0.00 & 0.18 & 2.65 & 2.88 & 3.00 & 3.13 & 3.36 & 1450 & 1.00\end{array}$

a_End[2] $\quad 3.29 \quad 0.000 .17 \quad 2.963 .18 \quad 3.303 .413 .6114331 .00$

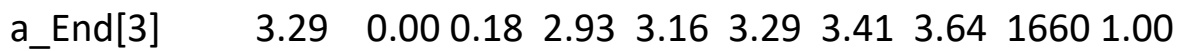

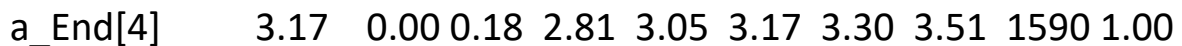

b_SES_SatIns $-0.16 \quad 0.000 .05-0.24-0.19-0.16-0.12-0.0615671 .00$

b_Insured_SatIns $-0.19 \quad 0.000 .05-0.29-0.23-0.19-0.15-0.0817021 .00$

b_Res_SatIns $\quad-0.50 \quad 0.000 .06-0.61-0.54-0.50-0.46-0.3916031 .00$

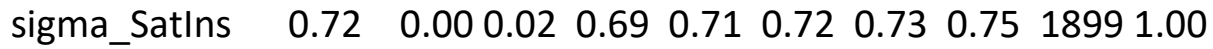

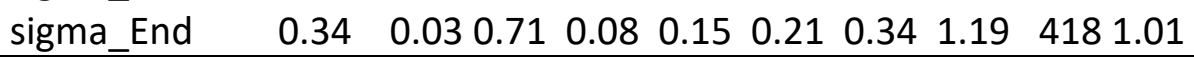

Chúng ta có thể thấy rằng effective sample size (n_eff) là ổn (trên 1000 thường là một dấu hiệu tốt), một số liệu chẩn đoán khác là giá trị Rhat cũng cho thấy sự hội tụ (Rhat bằng 1 là một dấu hiệu tốt, hơn 1.1 có thể chỉ ra là có vấn đề).

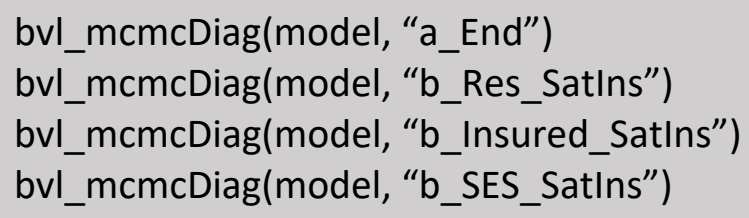

Chúng ta có được các đồ thị kiểm tra quy cách mô phỏng cho bộ hệ số b_SES_SatIns và b_Insured_SatIns và b_Insured_SatIns như sau: 


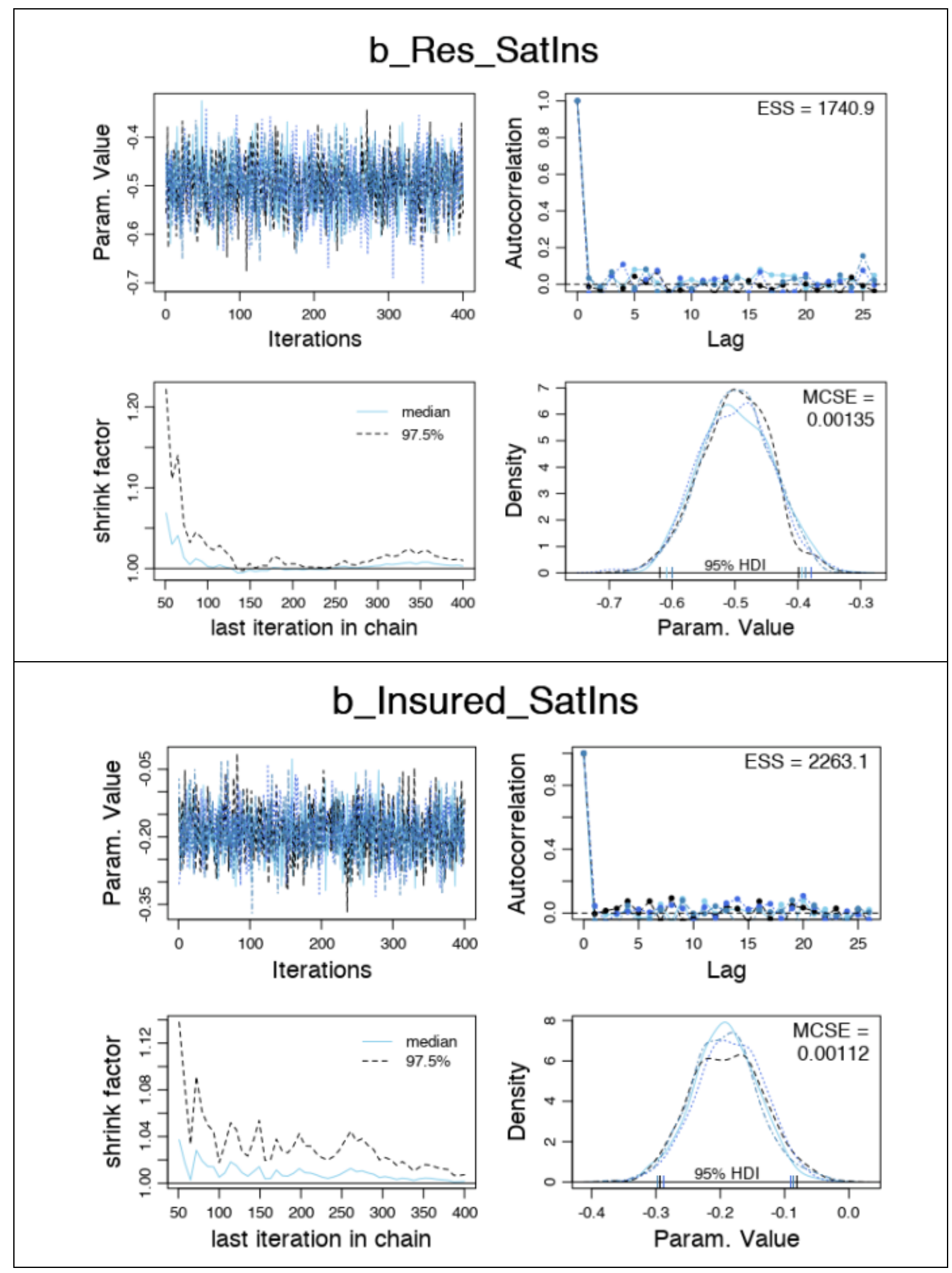




\section{b_SES_SatIns}
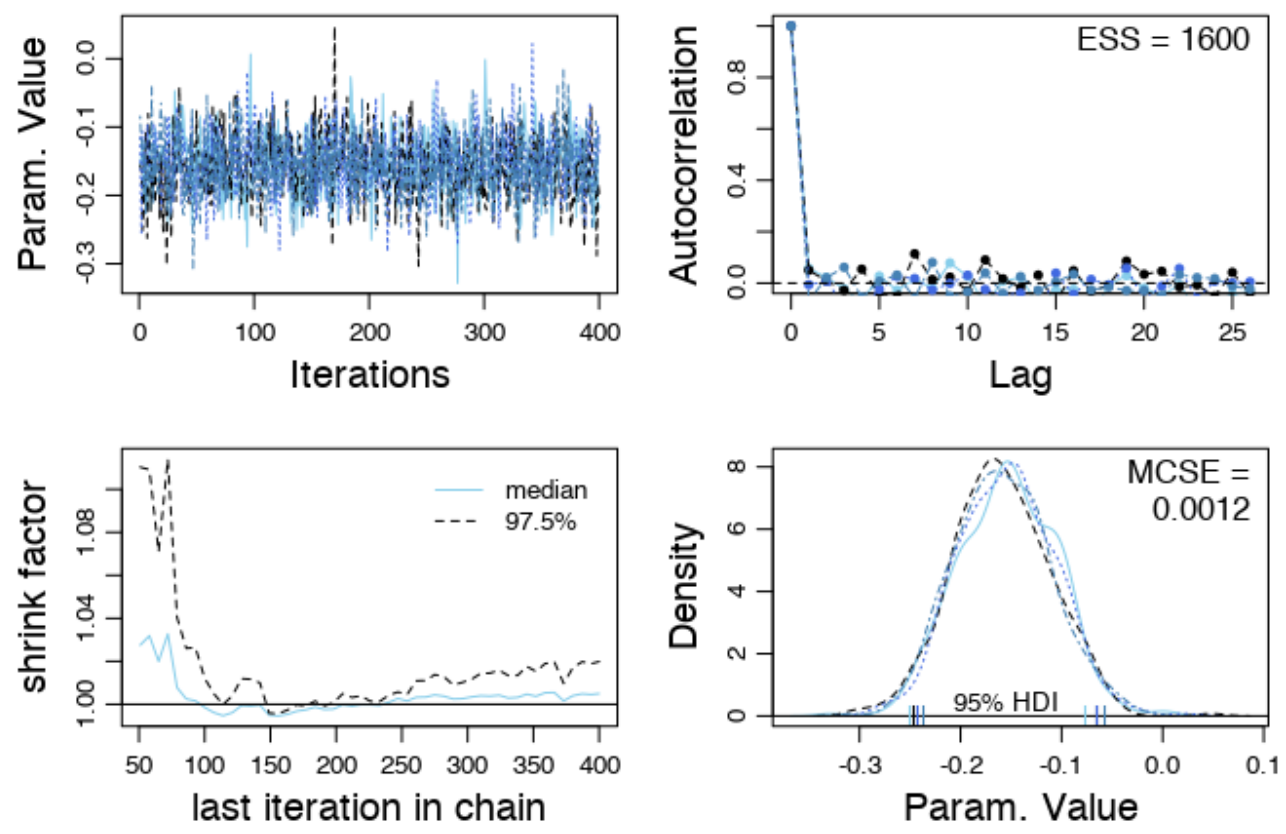

Riêng vector hệ số a_End[i] với i=1,2,3,4 được tách ra trong các đồ họa dưới đây:
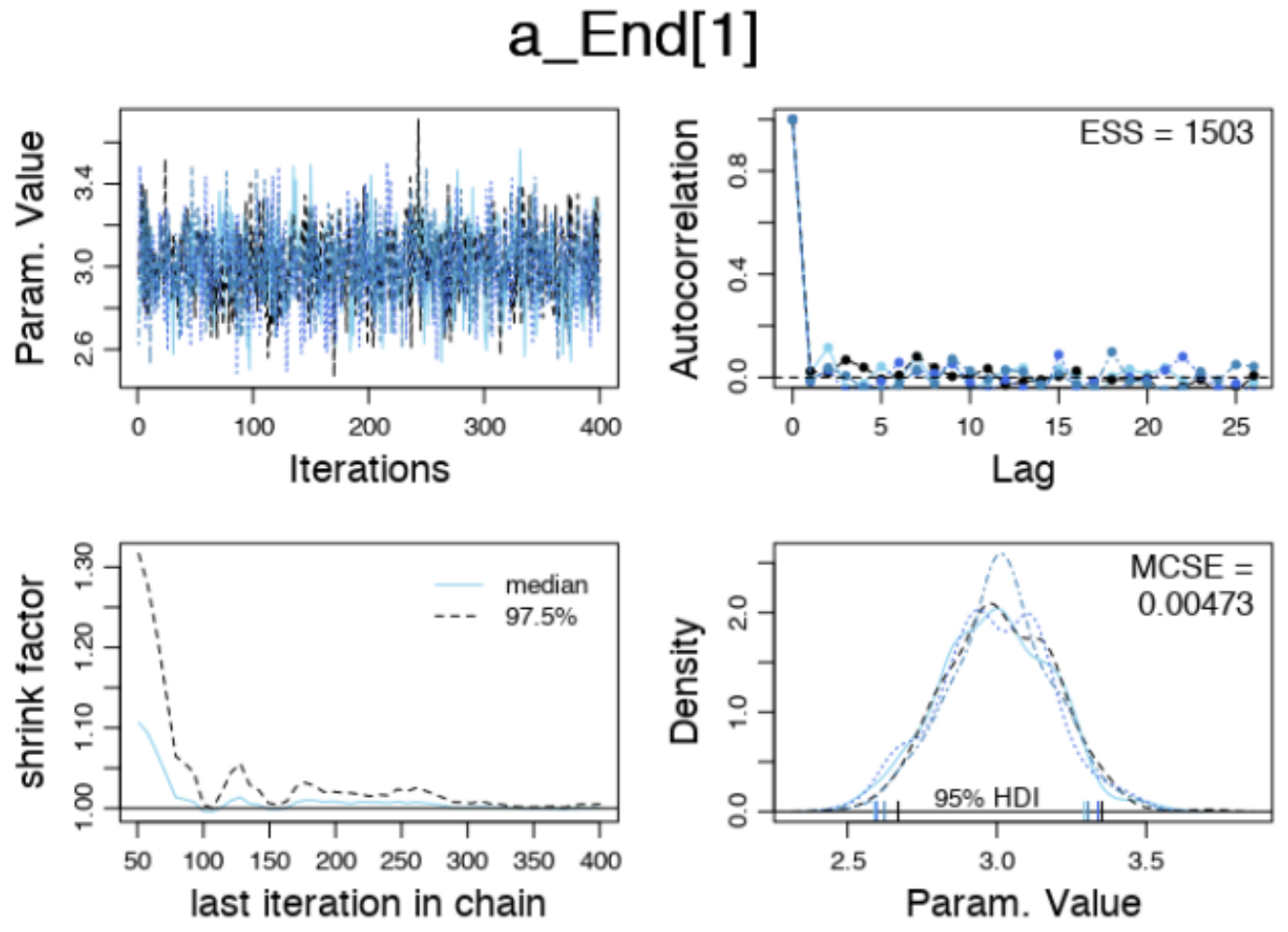


\section{a_End[2]}
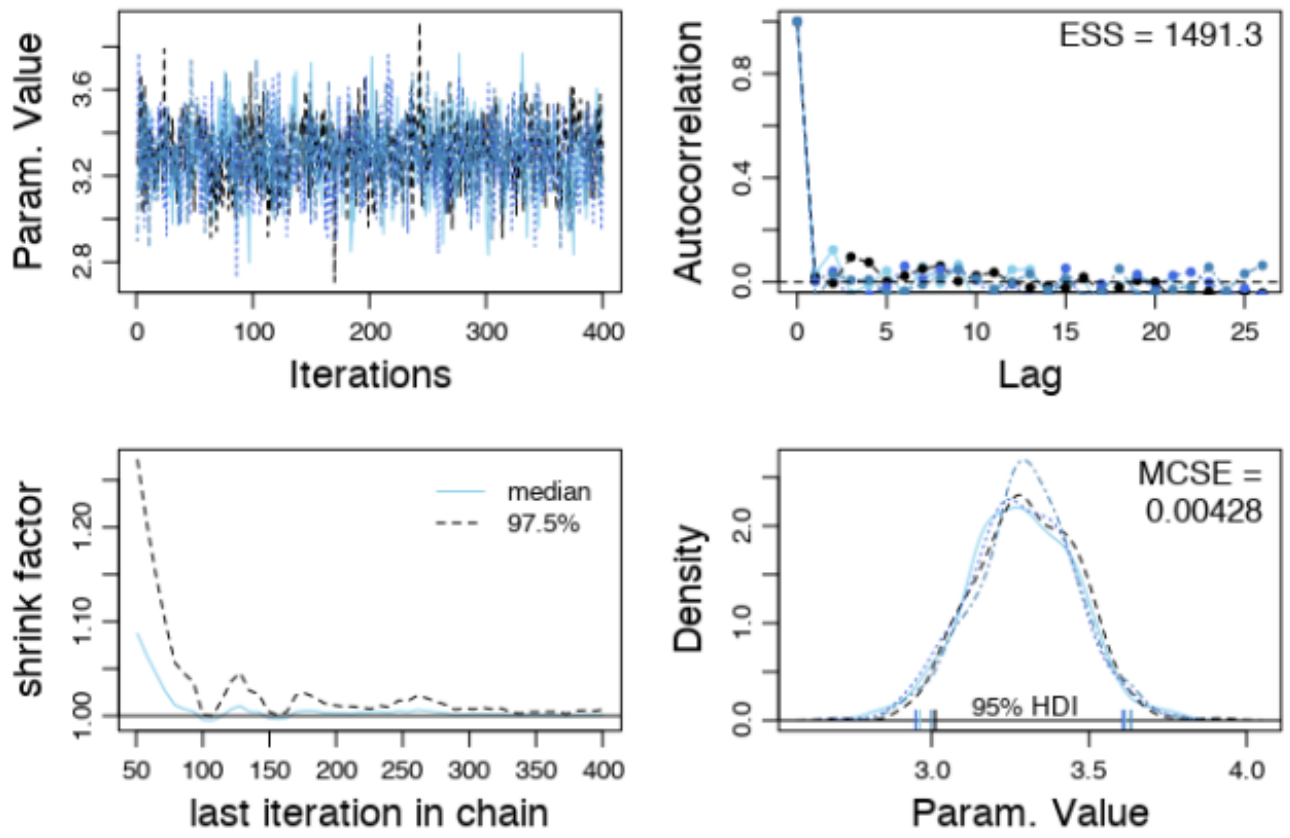

\section{a_End[3]}
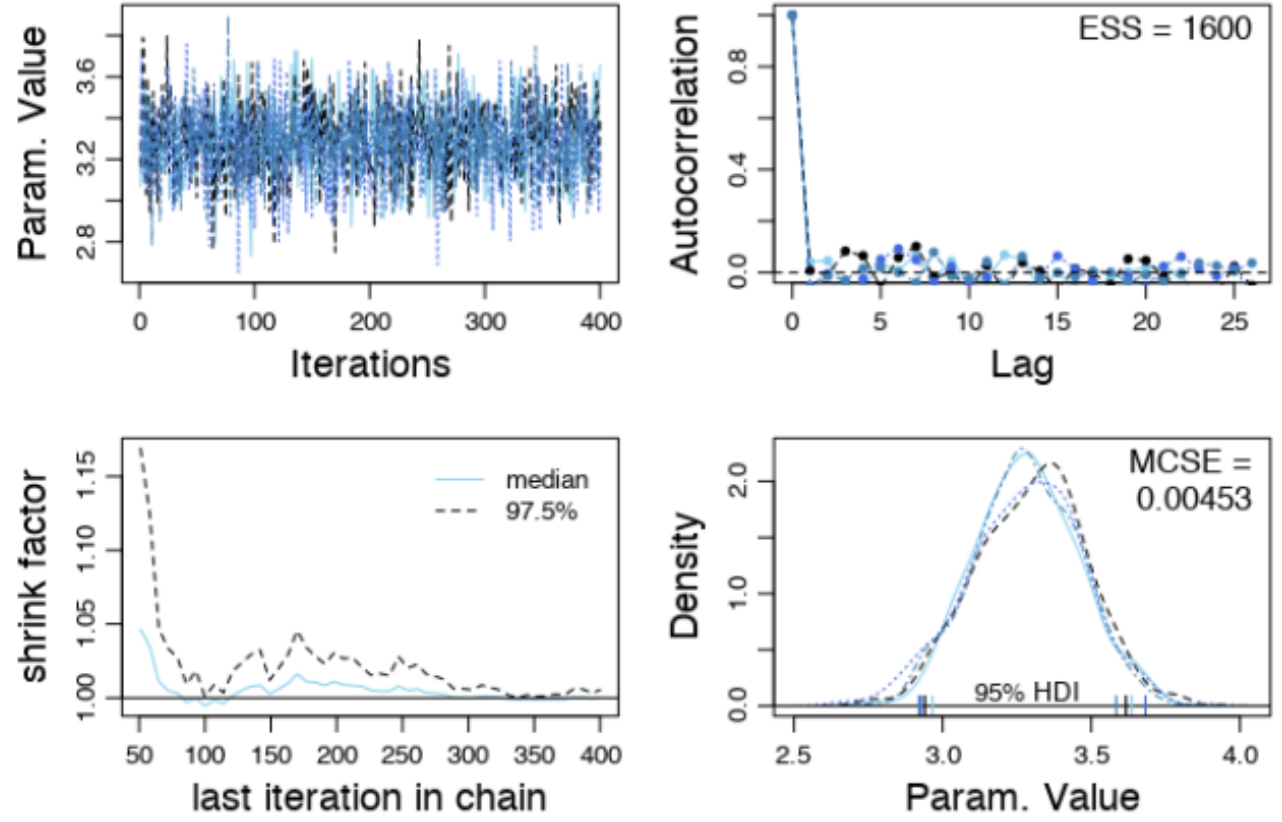


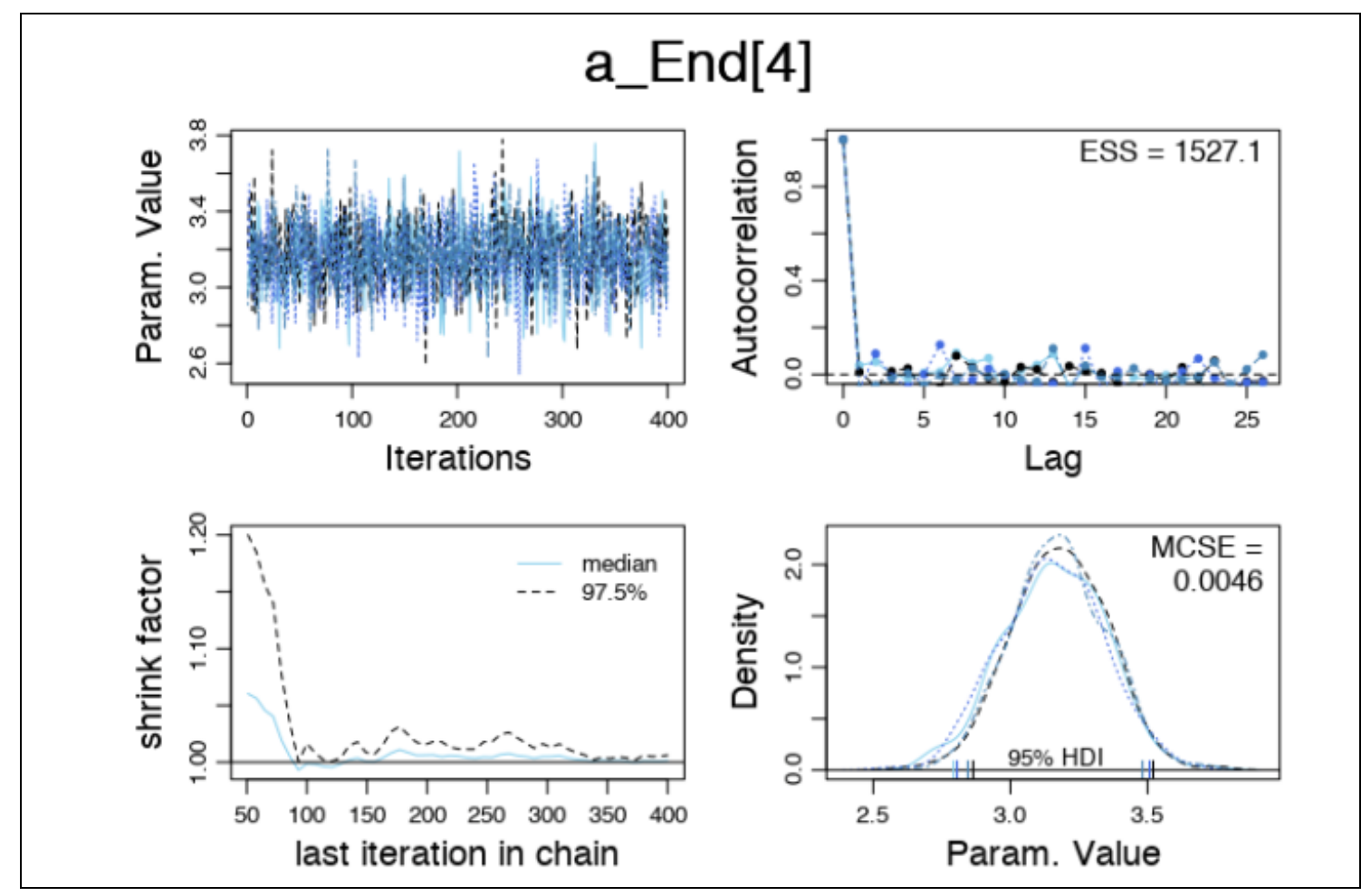

Việc kiểm tra tính hợp lệ, tốc độ hội tụ của xích, Rhat và eff có thể tiến hành hoàn toàn bình thường.

Nếu vẽ log posterior:

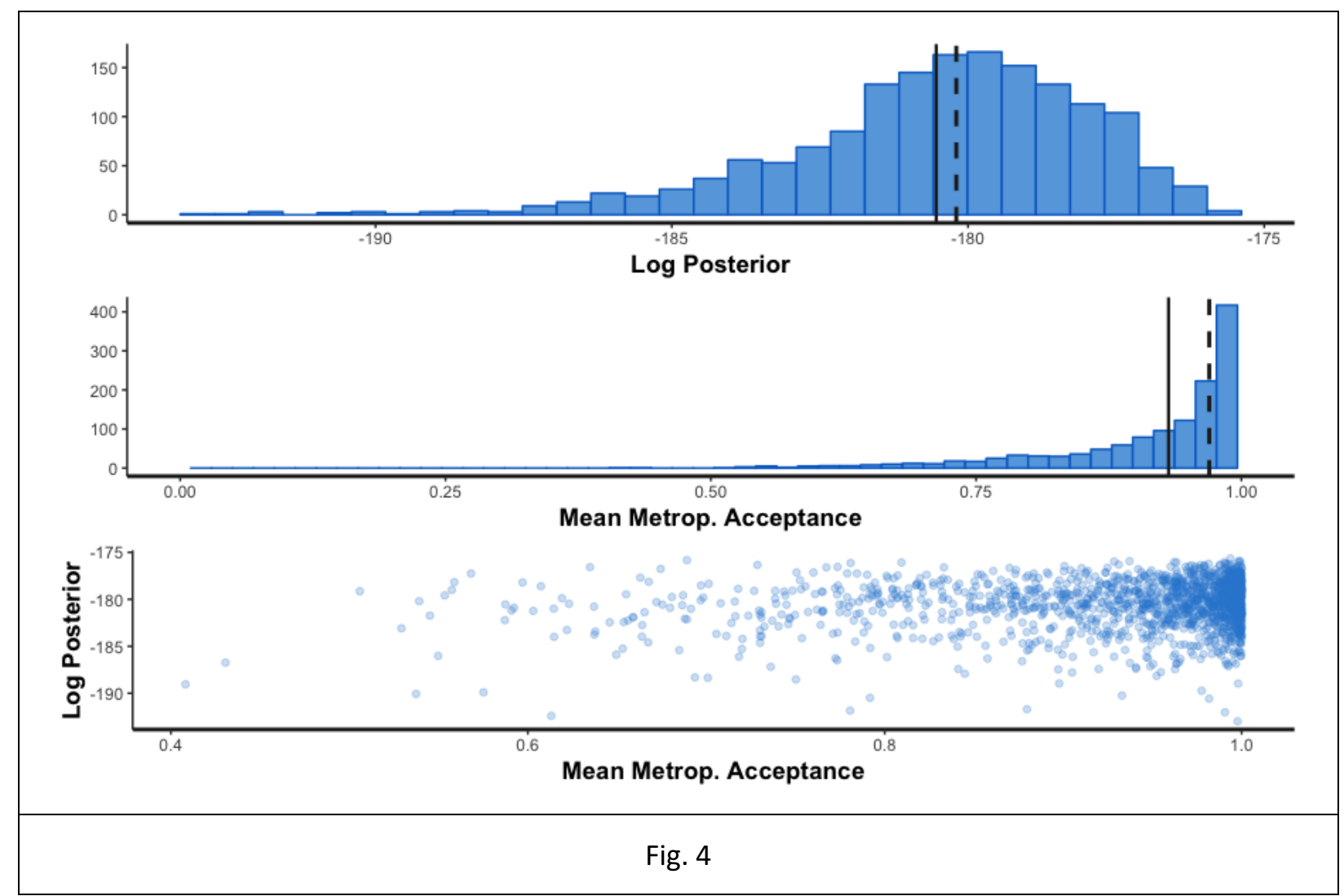


Đến đây, các kiểm tra posterior samples cơ bản hoàn thành. Trên phương diện kỹ thuật thống kê, mô hình có thể coi là đáp ứng được các yêu cầu (satisfactory).
e. Kết quả hồi quy

Các phân phối của sigma sau mô phỏng:

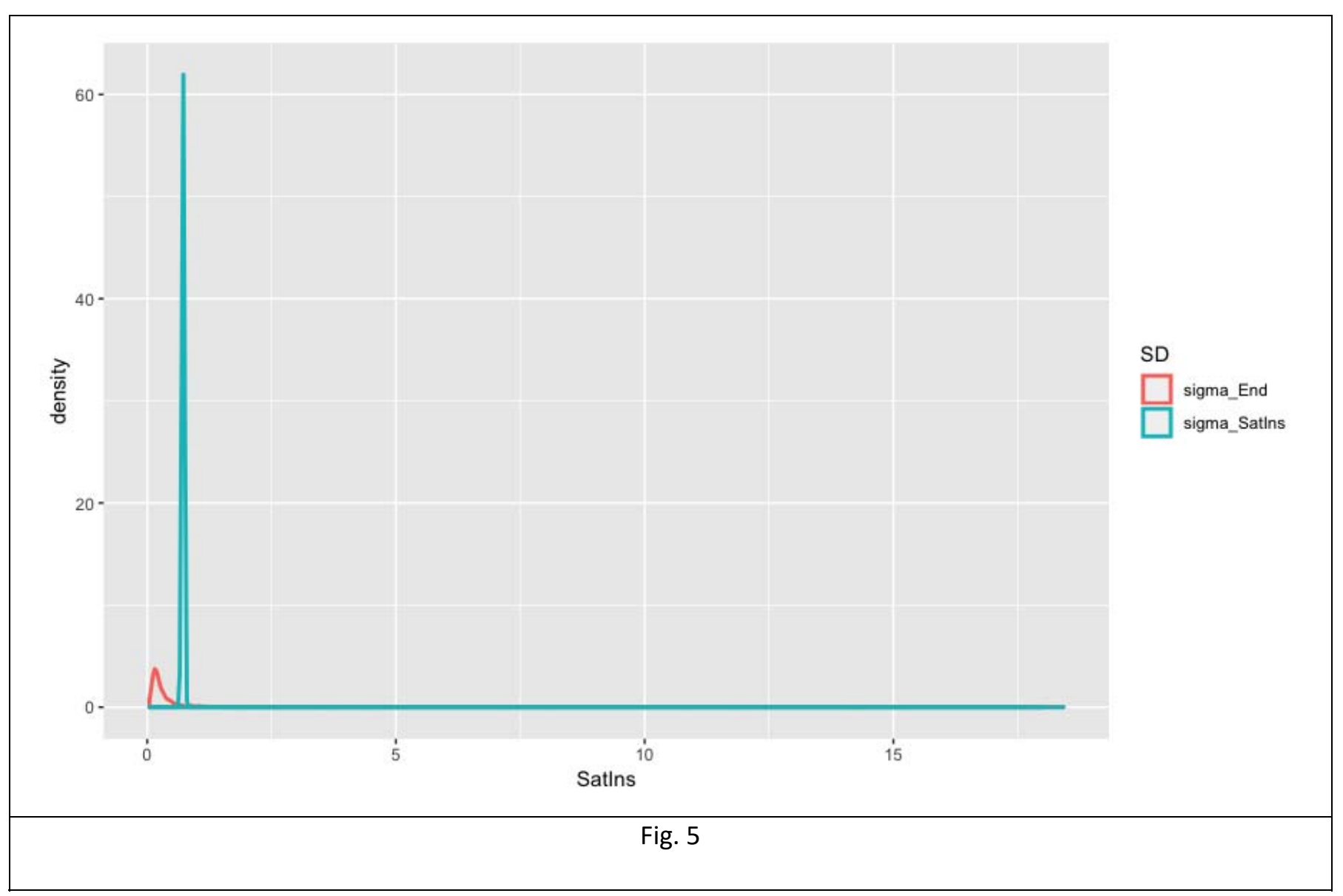

Phân phối các hệ số (Fig. 5):

bvl_plotParams(fit, 2, 2, 0.89)

Nếu vẽ interval phân phối với mức đánh giá 80\% (Fig. 6)

bvl_plotIntervals(fit, prob $=0.8$, prob_outer $=0.95$, color_scheme $=$ "brightblue" $)$

Figure 6: 


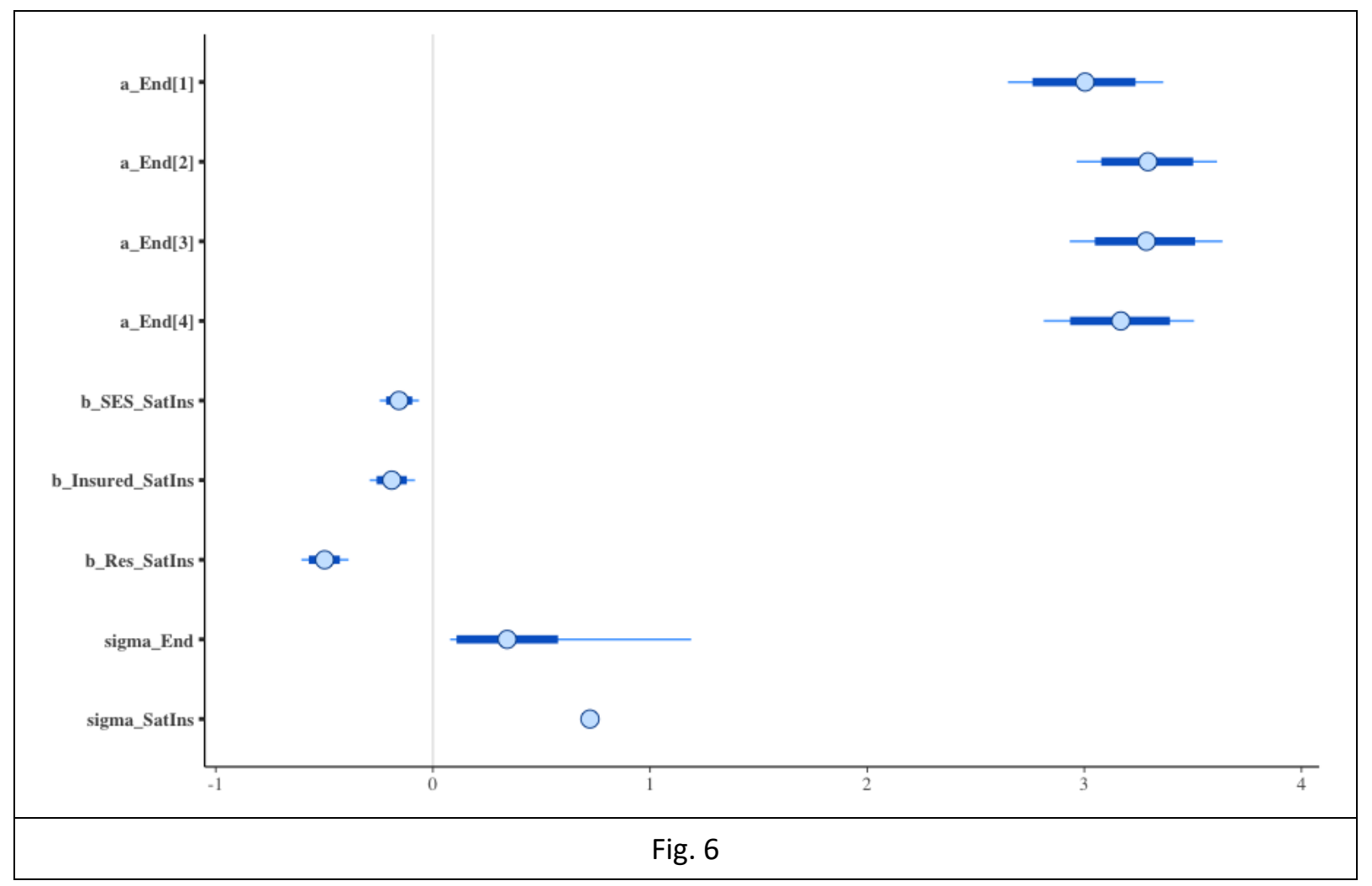

Tương ứng các cặp hệ số (Fig. 7):

\section{bvl_plotPair(fit, cex=1)}

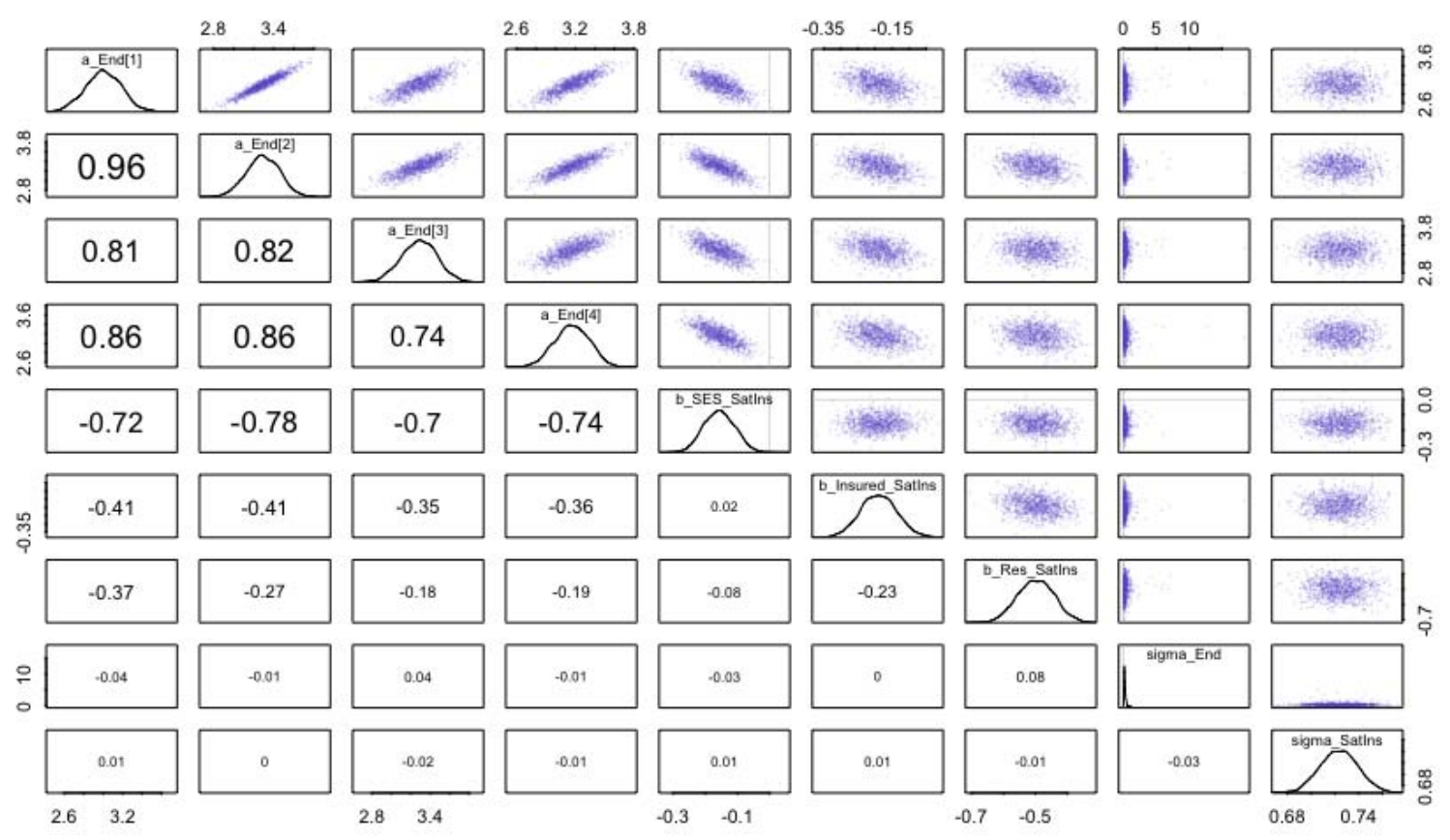

Fig. 7 
Nếu so sánh riêng 2 tham số b_Res, b_Insured:

ggplot(model@posterior, aes(x=(b_Insured_SatIns),y=(b_Res_SatIns), color = sigma_SatIns $))+$ geom_point(alpha $=0.3)+$

geom_density $2 \mathrm{~d}($ color $=$ "gray $30 ")+$

scale_color_viridis(option = "C")+

geom_abline(intercept=0, slope $=1$ )

Ba đồ họa quan hệ các cặp hệ số thu được dưới đây:

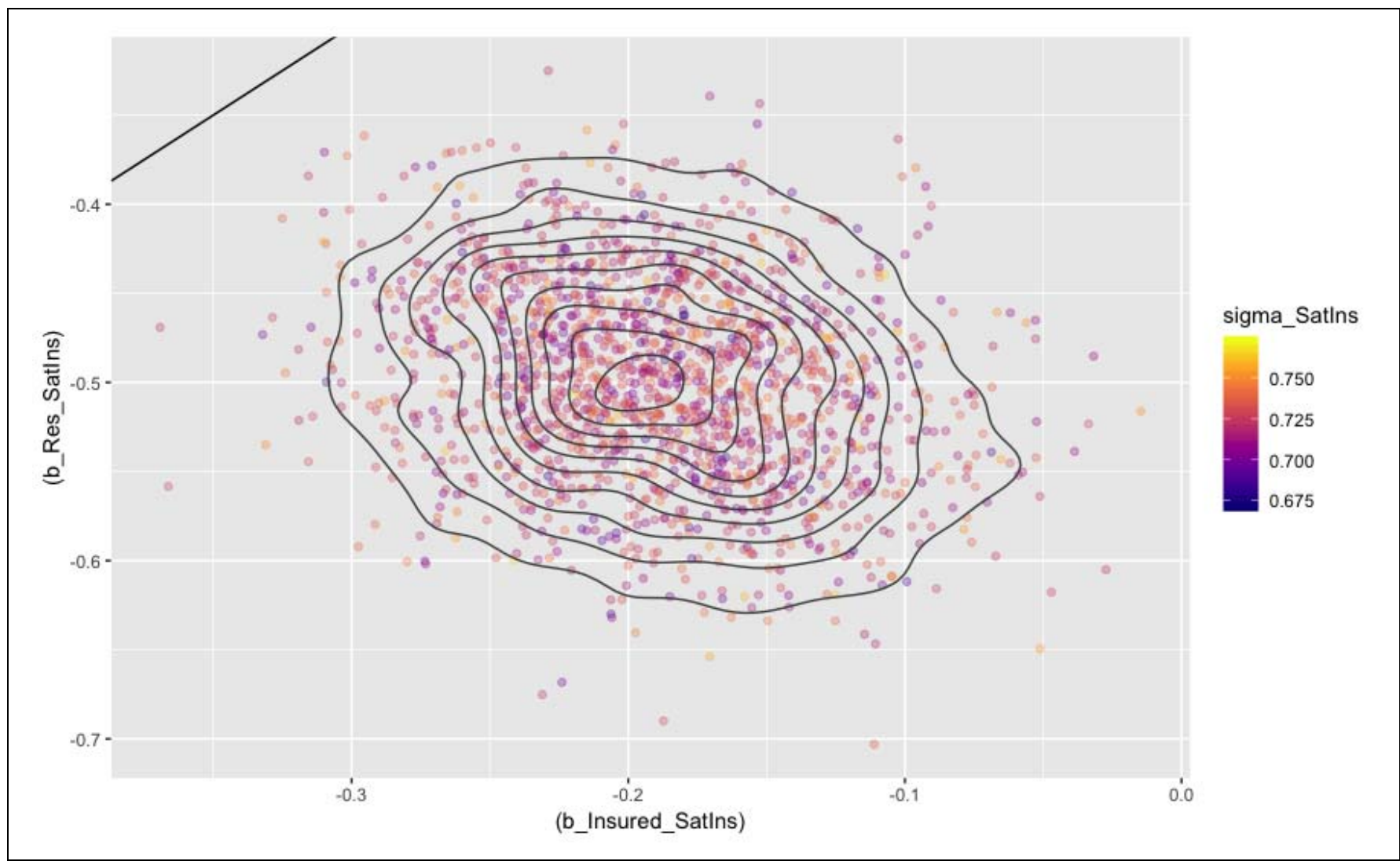

Fig. 8a 

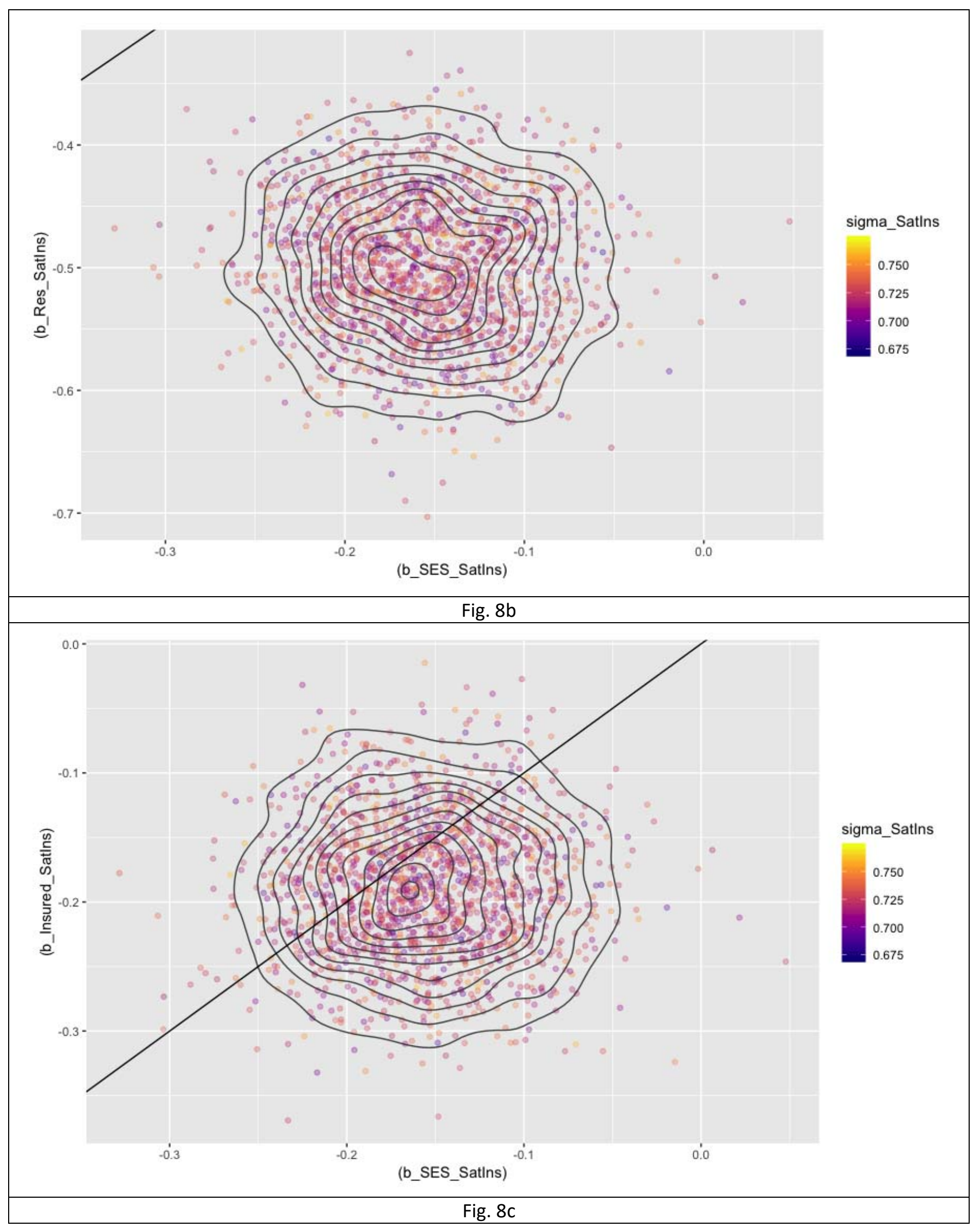
Các hình thuộc Figure 8 rất đáng chú ý và cần giải thích kỹ lưỡng. Chúng cũng có giá trị biện luận (logic) rất tốt, đặc trưng cho phương pháp Bayesian. Một vài ý giải thích cơ bản như sau.

Trên hình (Fig. 8a) ta thấy hệ số nghiêng nhiều về Res. Ảnh hưởng Res lên SatIns mạnh hơn so với insured. Tuy nhiên 2 hệ số này cùng dấu (-) ảnh hưởng cùng xu hướng.

Trên hình (Fig. 8b). Ảnh hưởng Res lên Satlns mạnh hơn so với SES. Tuy nhiên 2 hệ số này cùng dấu (-) ảnh hưởng cùng xu hướng.

Trên hình (Fig. 8c). Ảnh hưởng SES và Insured lên SatIns gần tương đương nhau, 2 hệ số này cùng dấu (-) ảnh hưởng cùng xu hướng.

Khôi phục lại phương trình hồi quy ta sẽ có 4 phương trình:

- Đối với các bệnh nhân khỏi bệnh End = A ta có:

$$
\text { SatIns }=3.00-0.16 * \text { SES }-0.50 * \text { Res }-0.19 * \text { Insured }
$$

- Đối với các bệnh nhân cần tiếp tục điều trị End = B ta có:

$$
\text { SatIns }=3.29-0.16 * \text { SES }-0.50 * \text { Res }-0.19 * \text { Insured }
$$

- Đối với các bệnh nhân dừng giữa chừng End = C ta có:

$$
\text { SatIns }=3.29-0.16 * \text { SES }-0.50 * \text { Res }-0.19 * \text { Insured }
$$

- Đối với các bệnh nhân kết thúc sớm End = D ta có:

$$
\text { SatIns }=3.17-0.16 * \text { SES }-0.50 * \text { Res }-0.19 * \text { Insured }
$$

So sánh hệ số alpha của 4 giá trị End được biểu diễn qua đồ thị dưới đây: 


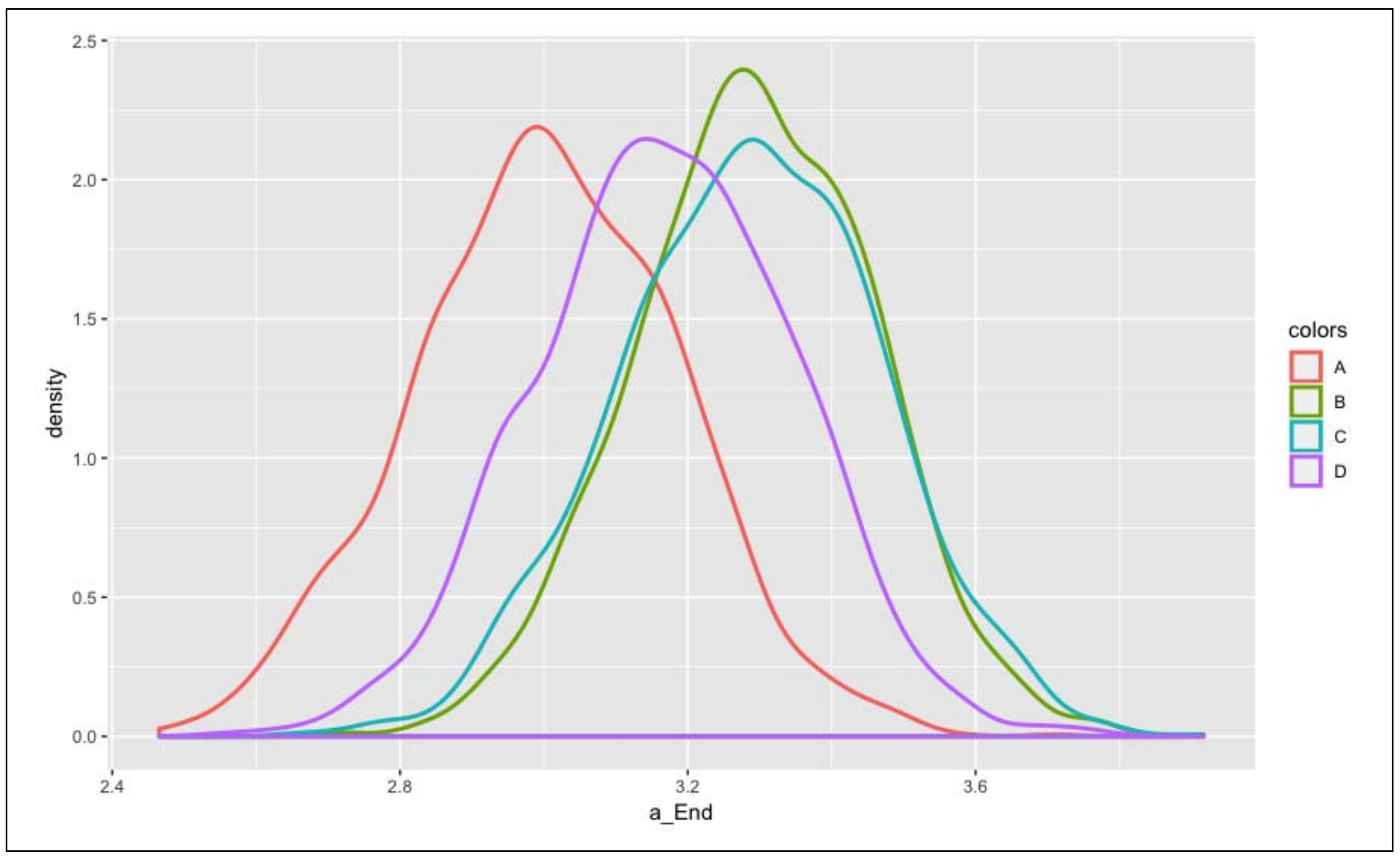

*Câu hỏi: Liệu có thể suy nghĩ gì khi dường như simulated data chỉ ra rằng:

“Những bệnh nhân khỏi bệnh ít hài lòng với dịch vụ nhất?!”

f. Posterior predictive checks

Kết quả y_rep sẽ cho ta thấy phân phối density distribution of the model predictions compare with that of the raw data

bvl_plotPPC(model, fun = "stat", stat = "mean", color_scheme = "blue")

Trong đồ thị hình Fig. 9 , ta thấy mean posterior (đường kẻ xanh) được align khá sát với raw data. 


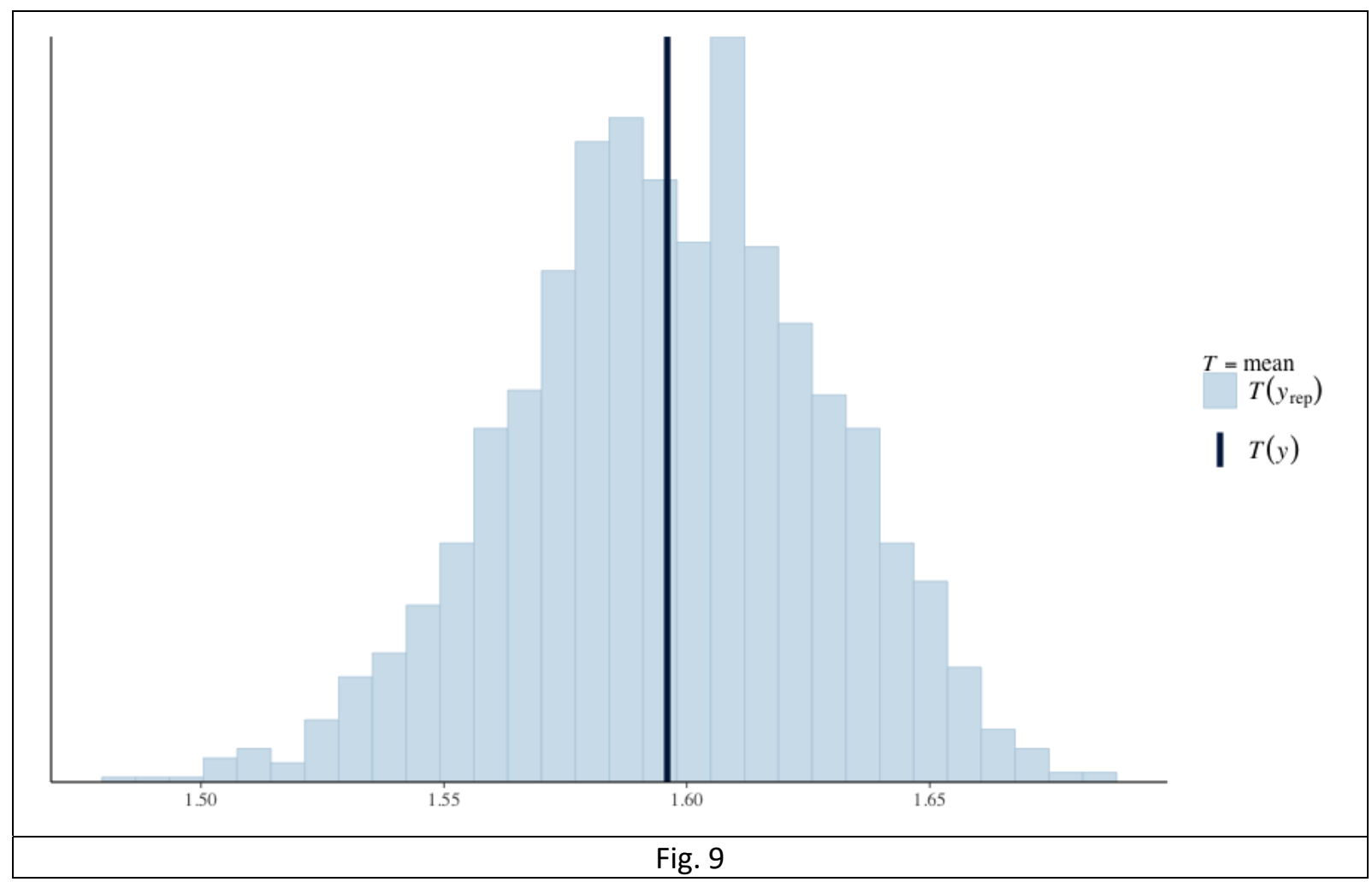

Nếu vẽ mật độ, ta có thể thấy phân phối (Fig. 10) như sau:

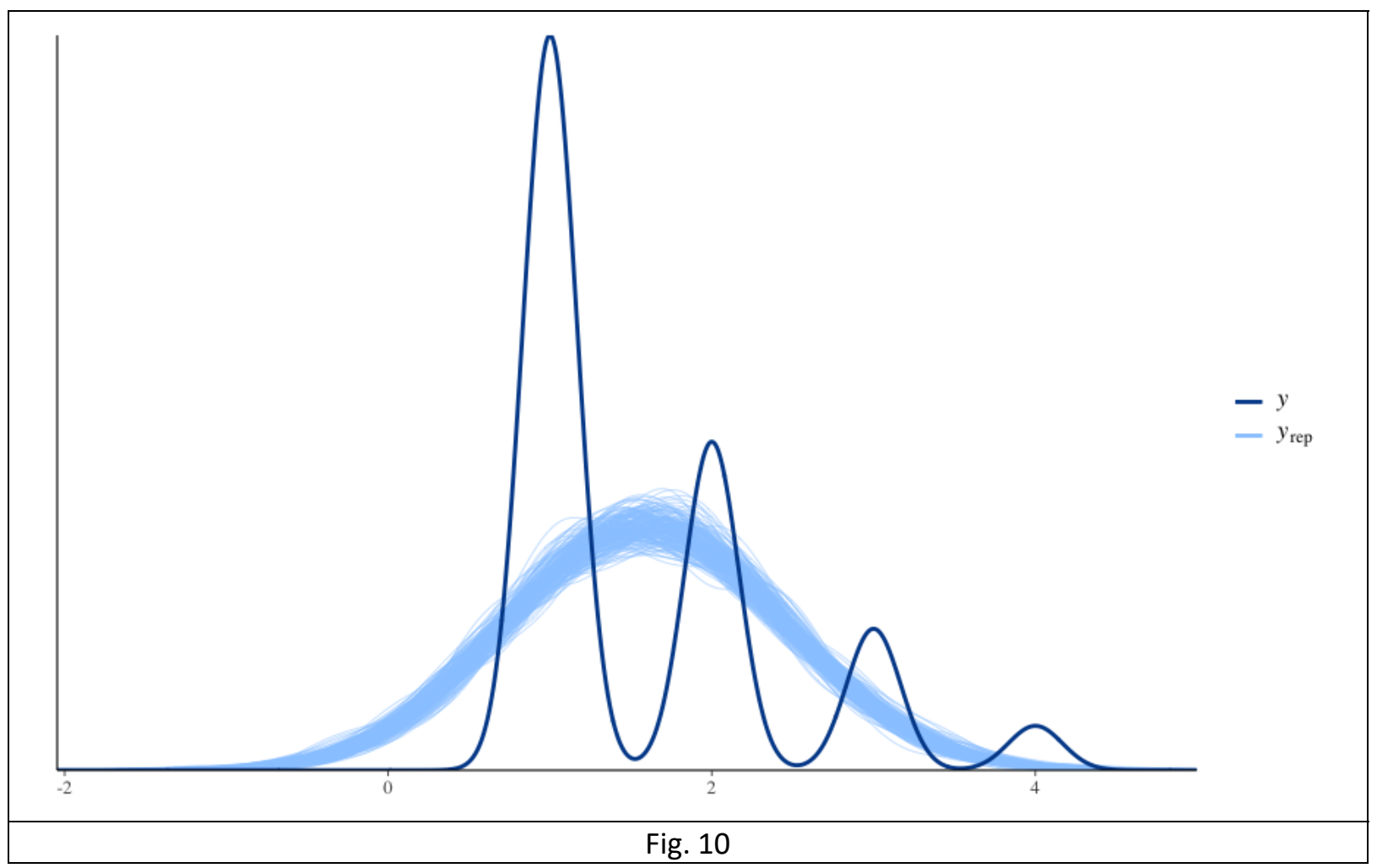

Cài thêm code để so sánh nhóm có bảo hiểm và không có bảo hiểm về mức độ hài lòng (đối với dịch vụ bảo hiểm Satıns): 


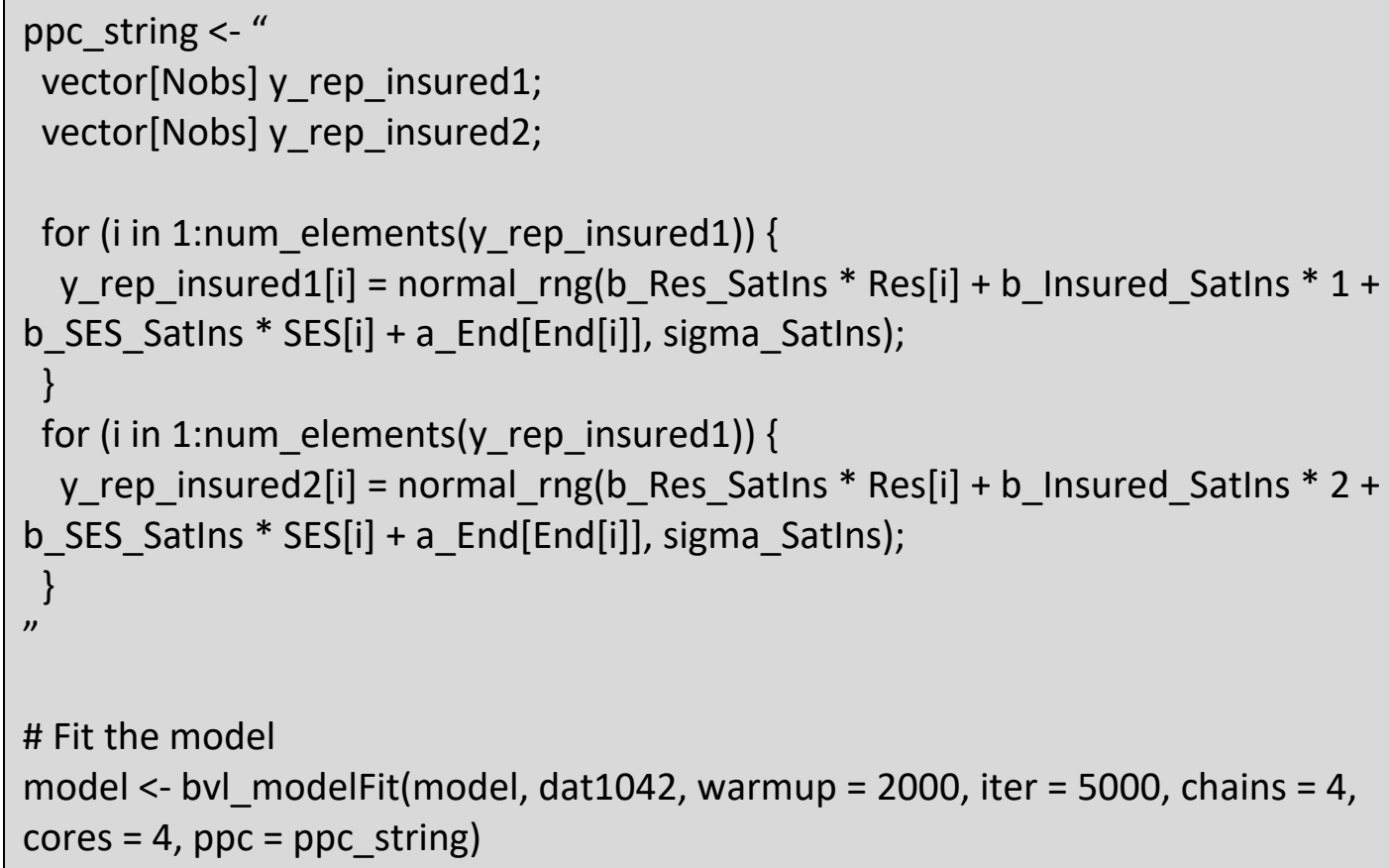

Trong phần code đưa thêm ppc_string ở trên, ta có chèn thêm 2 biến y_rep_insured1 và y_rep_insured2 để đánh giá kết quả trong 2 trường hợp bệnh nhân có và không bảo hiểm.

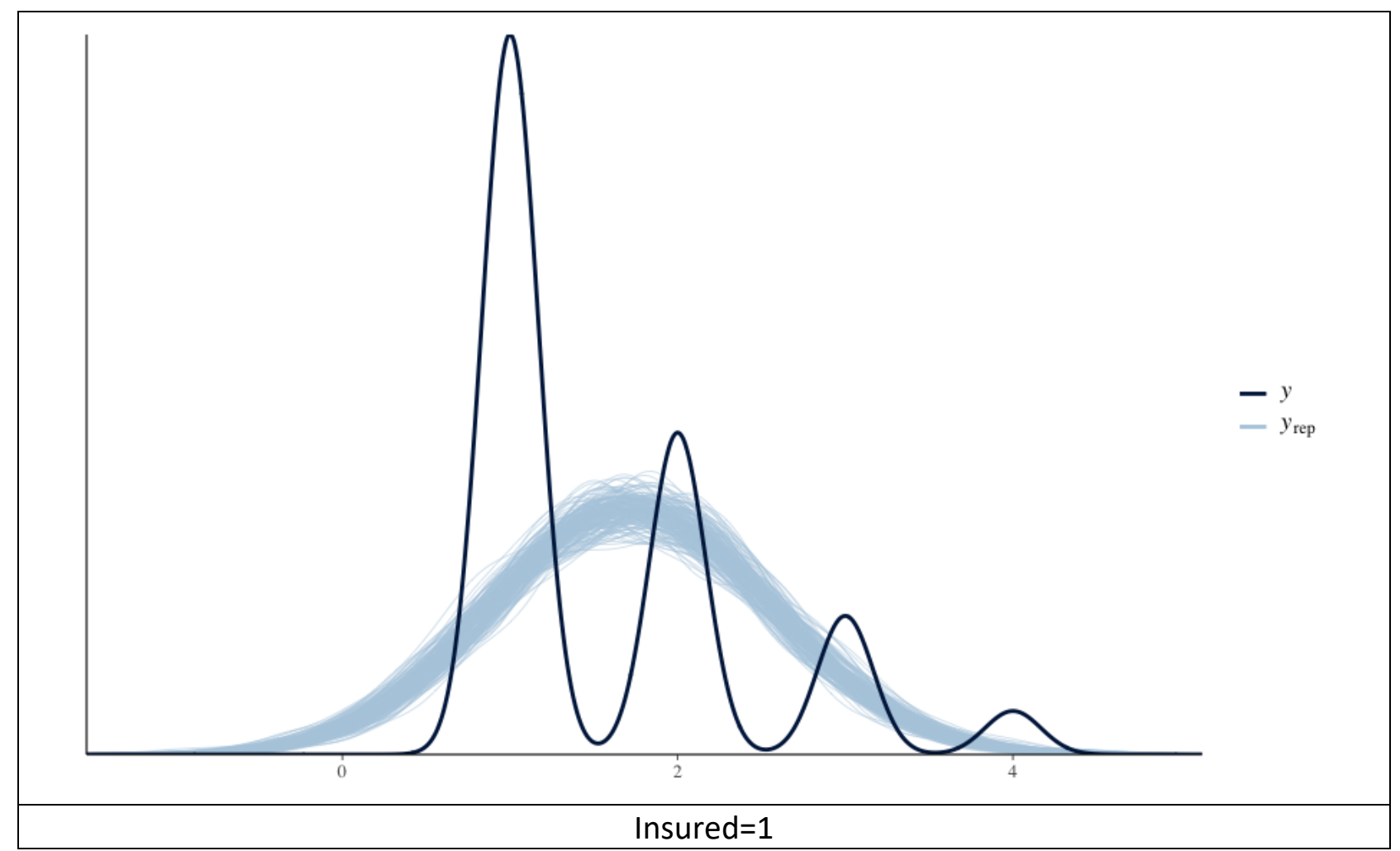




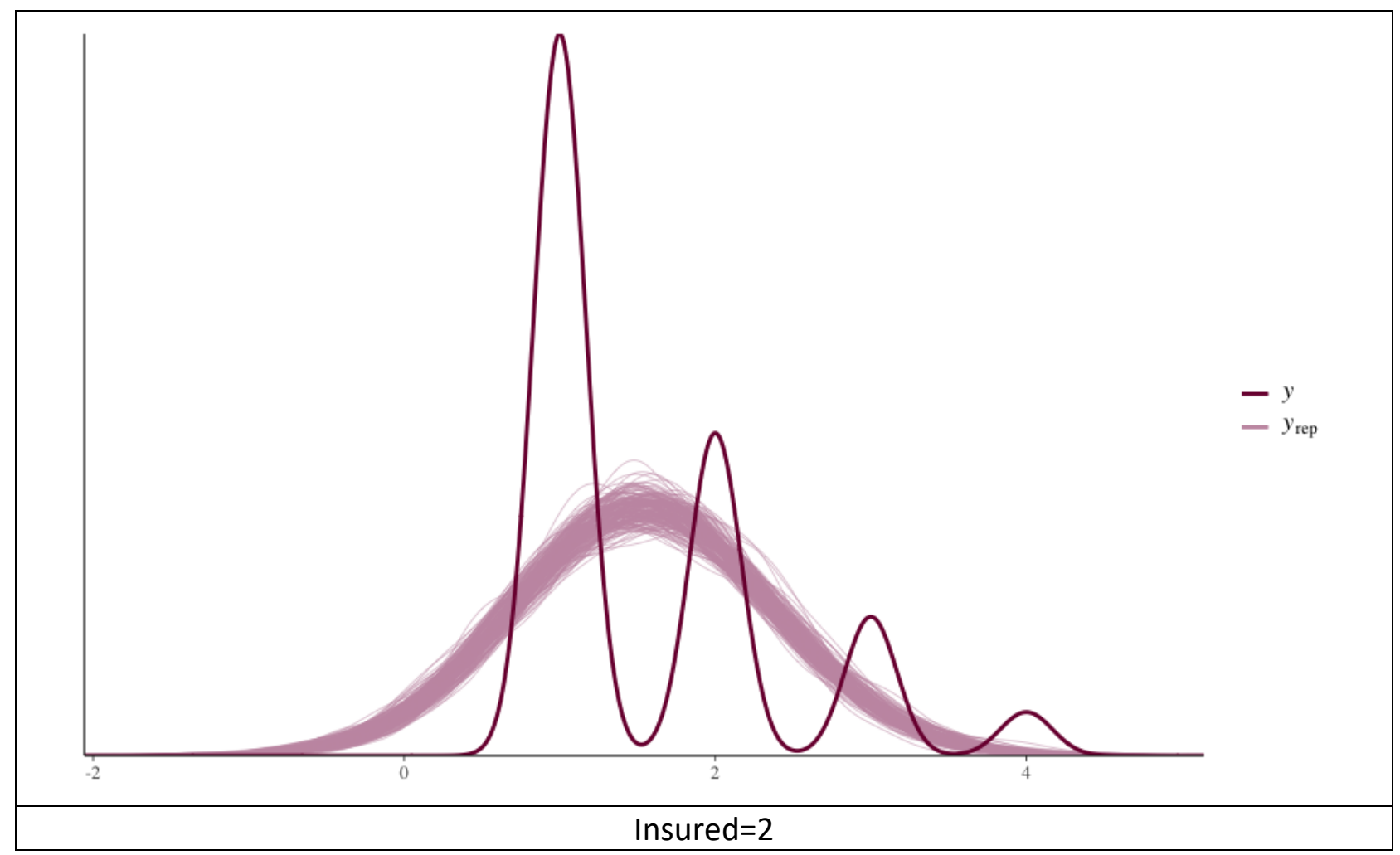

*Câu hỏi: Liệu có thể đưa ra đánh giá gì từ simulated data khi thực tế kết quả cho thấy: Dường như có bảo hiểm và không có bảo không làm người bệnh hài lòng hơn bao nhiêu?!

Phần Note $2 b$ cần được ghép với kết quả ở Note 2a [4], triển khai giải thích từ đơn giản đến phức tạp, để hoàn thiện một bức tranh chung về nghiên cứu. Các phát triển tiếp theo của BayesVL sẽ tìm hiểu tính thân thiện của rstanarm [7-8] để bổ sung tính năng, đặc biệt đồ họa và thư viện hàm dễ sử dụng.

\section{References:}

[1] Vuong QH, \& La VP. (2019). BayesVL package for Bayesian statistical analyses in R. Github: BayesVL version 0.6.5; DOI: 10.31219/osf.io/ya9u6. Available from: $<$ https://github.com/sshpa/bayesvl>.

[2] Vuong QH. (2015). Be rich or don't be sick: estimating Vietnamese patients' risk of falling into destitution. SpringerPlus, 4(1), 529. DOI: 10.1186/s40064-0151279-x.

[3] Ho MT, La VP, Nguyen MH, Vuong TT, Nghiem KCP, Tran T, Nguyen HKT, \& Vuong QH. (2019). Health care, medical insurance, and economic destitution: A dataset of 1042 stories. Data, 4, 57. DOI: 10.3390/data4020057.

[4] Vuong QH, \& La VP. (2019). Ứng dụng BayesVL v0.6.5 mô phỏng MCMC với bài toán burden res + insured sử dụng dữ liệu thực 1042 quan sát. OSF Preprint; DOI: 10.31219/osf.io/9rhyk. Available from: <https://osf.io/9rhyk/>. 
[5] McElreath R. (2018). Statistical Rethinking: A Bayesian Course with Examples in R and Stan. London: Chapman and Hall/CRC.

[6] Scutari M. (2010). Learning Bayesian networks with the bnlearn R package. Journal of Statistical Software, 35(3), 1-22.

[7] Muth C, Oravecz Z, \& Gabry J. (2018). User-friendly Bayesian regression modeling: A tutorial with rstanarm and shinystan. Quantitative Methods for Psychology, 14(2), 99-119.

[8] Gabry J, \& Goodrich B. (2016). rstanarm: Bayesian applied regression modeling via Stan. R package version 2.10.0. Available from: <https://cran.rproject.org/web/packages/rstanarm/index.html> 